\title{
Recycling Mitsunobu coupling: a shortcut for troublesome esterifications
}

\author{
Boudjema Menhour ${ }^{\mathrm{a}}$, Firmin Obounou Akong ${ }^{\mathrm{a}}$, Patrick Mayon ${ }^{\mathrm{a}}$, Karen Plé ${ }^{\mathrm{b}}$, \\ Sandrine Bouquillon ${ }^{\mathrm{a}}$, Stéphan Dorey ${ }^{\mathrm{c}}$, Christophe Clément ${ }^{\mathrm{c}}$, Magali Deleu ${ }^{\mathrm{d}}$, \\ Dominique Harakat ${ }^{\mathrm{e}}$, Arnaud Haudrechy ${ }^{\mathrm{a},}$ \\ anstitut de Chimie Moléculaire de Reims, UMR CNRS 7312, Université de Reims, 51687 Reims Cedex, France \\ ${ }^{\mathrm{b}}$ Institut de Chimie Organique et Analytique, UMR CNRS 7311, Université d'Orléans, 45067 Orléans Cedex, France \\ ${ }^{c}$ Unité de Recherche Vignes et Vins de Champagne, EA 4707, Université de Reims, 51687 Reims Cedex, France \\ ${ }^{\mathrm{d}}$ Laboratoire de Biophysique Moléculaire aux Interfaces, Université de Liège, Passage des Déportés, 2, 5030 Gemboux, Belgium \\ ' Service commun d'analyses, Institut de Chimie Moléculaire de Reims, UMR CNRS 7312, Université de Reims, 51687 Reims Cedex, France
}

\section{A R T I C L E I N F O}

\section{Article history:}

Received 6 September 2016

Received in revised form 22 September 2016

Accepted 28 September 2016

Available online 29 September 2016

Keywords:

Mitsunobu reaction

Rhamnolipids

One-pot two-step cross-metathesis-

reduction

\begin{abstract}
A B S T R A C T
An unusual recycling Mitsunobu reaction proved to be successful to couple two fragments in the course of the synthesis of the hydrophobic moiety of rhamnolipid derivatives. Based on the obtained pivotal intermediate, a one pot 'cross-metathesis/reduction' approach gave access to structural variations of the side chains. Further study of these molecules will contribute to a better understanding of the role of the lipid moiety in immunostimulatory and plant defense eliciting properties.
\end{abstract}

(C) 2016 Elsevier Ltd. All rights reserved.

\section{Introduction}

Reviewing the literature has shown that Mitsunobu reactions ${ }^{1}$ with allylic alcohols can be performed with saturated carboxylic acids, giving a clean inversion of configuration. Examples with formic acid and two carbon-'acetic acid derivatives' have been described but we wish to focus our attention on particularly interesting cases with more complex carboxylic acid derivatives (with at least three carbons). ${ }^{2}$

In the literature, benzene, THF and toluene are the solvents of choice with diethyl ether, methylene chloride, DME or acetonitrile being used more rarely. ${ }^{2 \mathrm{j}, 3}$

In the course of our studies on bacterial rhamnolipids, ${ }^{4}$ especially those produced by Pseudomonas aeruginosa ${ }^{5}$ and Burkholderia plantarii, ${ }^{6}$ all of our attempts to couple $\mathbf{1}(\boldsymbol{S})$ and $\mathbf{2}(\boldsymbol{S})$ via diverse esterification procedures were unsuccessful (DCC/DMAP, EDCI and TBTU).

\footnotetext{
* Corresponding author. E-mail address: arnaud.haudrechy@univ-reims.fr (A. Haudrechy).
}

Surprisingly, compound $\mathbf{3}(\boldsymbol{S})$ was the only observed product resulting from intermolecular acetate migration of the DCC intermediate as previously described by Duynstee et al. ${ }^{8}$

\section{Results and discussion}

Our starting material, acetoxyester $\mathbf{3}(\boldsymbol{S}),{ }^{9}$ was easily prepared on a convenient scale using PS Amano lipase as a biocatalyst for the enantioselective acetylation (Scheme 2). ${ }^{10}$ Loss of half of the starting material, inherent in an enzymatic resolution was an initial drawback, but this fact later proved beneficial.

While attempting the various coupling reactions, work was also in progress to recycle the unwanted enantiomer $\mathbf{1}(\boldsymbol{R})$ via a Mitsunobu approach.

The idea was to perform the Mitsunobu reaction between diverse carboxylic acids derived from the acetoxyester $\mathbf{3}(\boldsymbol{S})$ and the allylic alcohol $\mathbf{1}(\boldsymbol{R})$, the undesired enantiomer, thus solving the problems of recycling and coupling in only one step.

Two preliminary tests gave very promising results in encouraging yields (Scheme 3 ). The first example allowed us to recycle the undesired enantiomer $\mathbf{1}(\boldsymbol{R})$ to give the $(\boldsymbol{S})$ intermediate $\mathbf{3}$, while the 


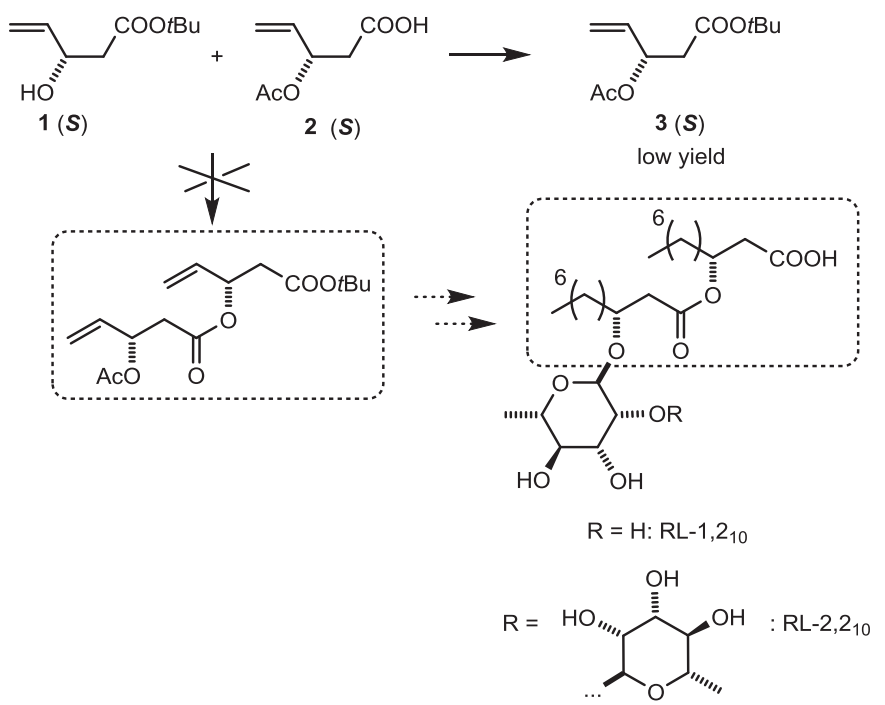

Scheme 1. Unsuccessful esterification to produce natural rhamnolipids used as elicitors.

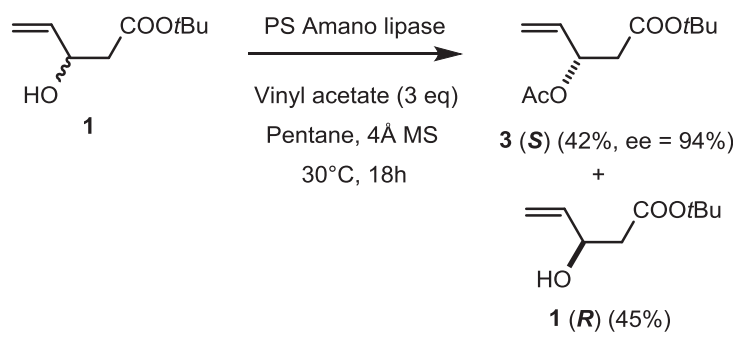

Scheme 2. Enzymatic resolution using PS Amano lipase.

second one solved two problems, the aforementioned desired inversion of configuration and coupling to furnish compound $\mathbf{4}$. This second example proved to be an esthetic alternative to the desired but unsuccessful DCC coupling reaction.

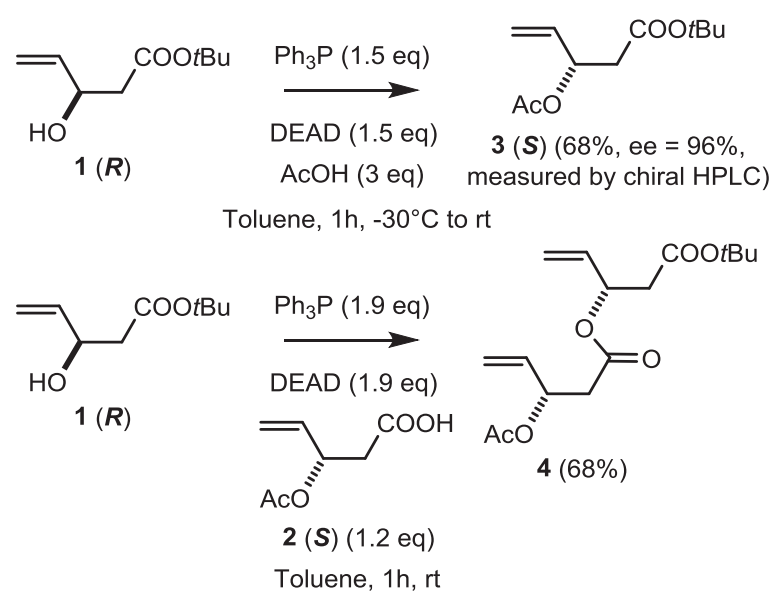

Scheme 3. Mitsunobu reactions on hydroxylester $\mathbf{1}(\boldsymbol{R})(1)$.

In order to apply this concept to longer chains carboxylic acids, attracted by the moieties involved in the natural Rhamnolipids (see Scheme 1), we used our pivotal substrate $\mathbf{3}(\boldsymbol{S})^{11}$ with 16 different alkenes to give the desired unsaturated long chain acids using conditions recently described by Voigtritter et al. (Table 1 ). ${ }^{12}$
Table 1

Cross-metatheses with compound $\mathbf{3}(\boldsymbol{S})$

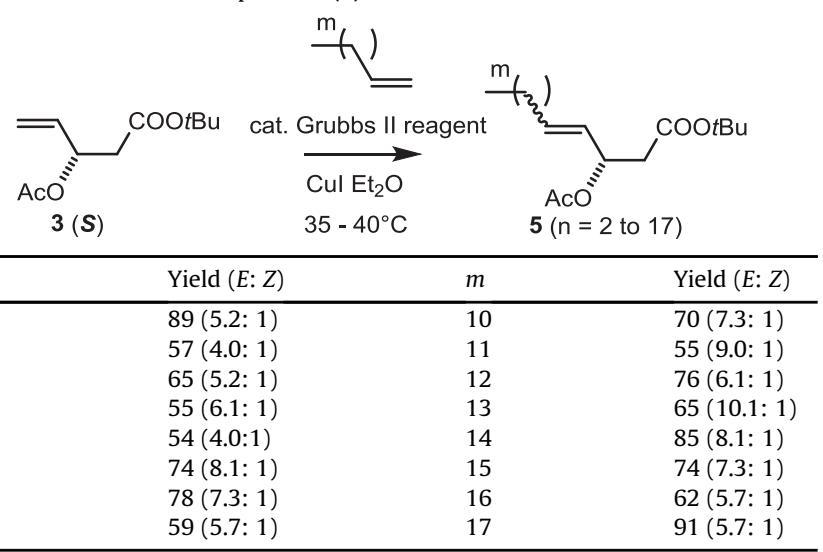

As the separation of $Z$ and $E$-alkenes proved to be difficult in certain cases, we decided to directly hydrogenate the crude mixture in a one-pot sequence (Scheme 4).

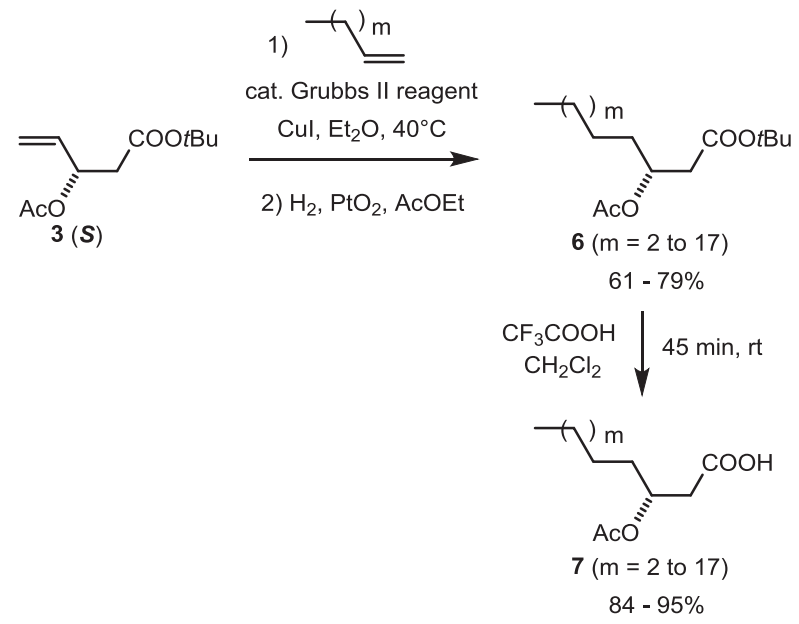

Scheme 4. Cross metathesis-hydrogenation of ester $\mathbf{3}(\boldsymbol{S})$

Compound 7 ( $m=2$ ) was then subjected to a Mitsunobu coupling with hydroxylester $\mathbf{1}(\boldsymbol{R})$ giving the expected compound $\mathbf{8}(m=2)$ in $58 \%$ yield (Scheme 5 ).

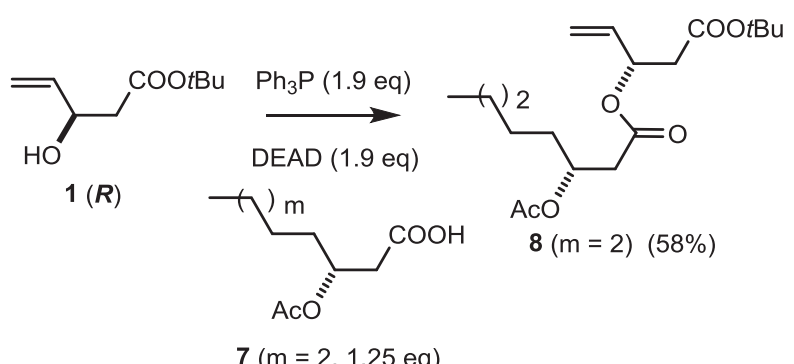

Toluene, $1 \mathrm{~h},-30^{\circ} \mathrm{C}$ to $\mathrm{rt}$

Scheme 5. Mitsunobu reactions with hydroxylester $\mathbf{1}(\boldsymbol{R})(2)$ 
Finally, to demonstrate the generality of our approach, a second cross-metathesis reaction was performed with derivative $\mathbf{8}(m=2)$ with five different alkenes, followed by direct hydrogenation of the reaction mixture (Table 2 ). In all cases, dimeric structures from the alkenes and approximately $15 \%$ of starting material could be easily removed by silica gel column chromatography. Acidic hydrolysis then gave the desired acids in yields varying between 70 and $88 \%$ yield.

\section{Table 2}

Cross metathesis of ester $\mathbf{8}(m=2)$

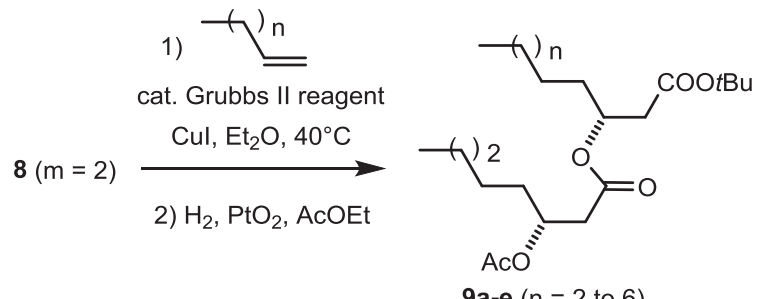

9a-e $(n=2$ to 6$)$
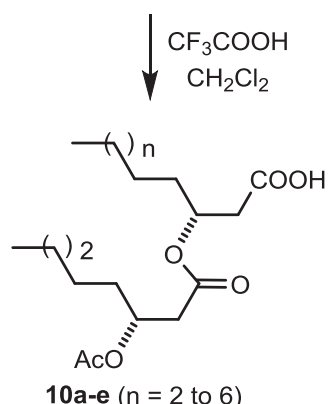

10a-e $(\mathrm{n}=2$ to 6$)$

\begin{tabular}{llllll}
\hline$n$ & 2 & 3 & 4 & 5 & 6 \\
\hline Metathesis yield 9a-e (\%) & 60 & 53 & 48 & 54 & 54 \\
Deprotection yield 10a-e (\%) & 76 & 86 & 70 & 73 & 88 \\
\hline
\end{tabular}

\section{Conclusion}

Using the Mitsunobu reaction for esterification allowed the recycling of an unwanted enantiomer with the simultaneous coupling of acid and alcohol fragments impossible to perform with more traditional coupling techniques, thus 'killing two birds with one stone'. This short and efficient strategy was effectively employed for the synthesis of the hydrophobic moiety of biologically important rhamnolipids. In our synthesis, two one-pot metathesis/hydrogenation sequences gave access to a large number of functionalized alkyl side chains. Work is in progress towards subsequent rhamnosylation which will allow us to obtain hybrid structures and a better understanding of the structure-activity relationships of this fascinating class of elicitors.

\section{Experimental section}

All reactions were carried out under argon and in oven-dried apparatus. Dry solvents were used in all experiments. Thin layer chromatography was performed on E. Merck pre-coated 60 F254 plates and compounds were observed by UV or by charring the plates with a phosphomolybdic acid system. Flash column chromatography (silica gel $40-63 \mu \mathrm{m}$ ) was carried out with light petroleum ether-ethyl acetate mixtures as eluent.

All the reported NMR spectra were recorded by dissolving the samples in $\mathrm{CDCl}_{3}$ (Eurisotop) on Bruker spectrometer (250, 500 or
$600 \mathrm{MHz}$ for $1 \mathrm{H}$ and 63,125 or $150 \mathrm{MHz}$ for $13 \mathrm{C}$ ) using standard pulse programs. Chemical shifts $(\delta)$ reported in parts per million $(\mathrm{ppm})$ refer to the chloroform peak of $\mathrm{CDCl}_{3}$ as reference at $7.26 \mathrm{ppm}$ and the coupling constants are reported in $\mathrm{Hz}$. The multiplicities are reported as follows: $\mathrm{br}=$ broad, $\mathrm{s}=$ singlet, $\mathrm{d}=$ doublet, $\mathrm{t}=$ triplet, $\mathrm{q}=$ quartet, $\mathrm{m}=$ multiplet. The chemical shifts of the ${ }^{13} \mathrm{C}$ NMR signals were assigned by using the center peak of the triplet of $\mathrm{CDCl}_{3}$ as reference $(\delta=77.16 \mathrm{ppm})$. FTIR spectra were recorded on Nicolet Avatar 320 FTIR films. Optical rotations were measured on a Perkin-Elmer 341 polarimeter. Electrospray ionization mass spectrometry experiments (MS and HRMS) were obtained on a hybrid tandem quadrupole/time of flight (Q-TOF) instrument, equipped with a pneumatically assisted electrospray (Z-spray) ion source (Micromass, Manchester, $\mathrm{UK})$ operated in positive mode $\left(\mathrm{eV}=30 \mathrm{~V}, 80^{\circ} \mathrm{C}\right.$, injection flow $5 \mu \mathrm{L} / \mathrm{min})$.

\section{1. (R) $t$-Butyl 3-hydroxy-pent-4-enoate 1 and $(S) t$-Butyl 3- acetoxy-pent-4-enoate 3}

Vinyl acetate ( $15.6 \mathrm{~mL}, 169.6 \mathrm{mmol}$ ) was added to a solution of $\mathbf{1}$ (9.75 g, $56.7 \mathrm{mmol})$ in pentane $(120 \mathrm{~mL}$ ). Amano lipase (from Burkholderia cepacia (6.2 g) and MS $4 \AA$ ( $8.9 \mathrm{~g})$ were added and the suspension was stirred at $30^{\circ} \mathrm{C}$ for $16 \mathrm{~h}$. The reaction mixture was monitored by TLC. The lipase and sieves were filtered and washed with $\mathrm{Et}_{2} \mathrm{O}$. The solvent was removed and the crude product was purified by silica gel column chromatography (EtOAc/Petroleum ether 1:9) to afford $\mathbf{1}(\boldsymbol{R})(4.4 \mathrm{~g}, 25.6 \mathrm{mmol}, 45 \%)$ and $\mathbf{3}(\boldsymbol{S})(5.1 \mathrm{~g}$, $23.8 \mathrm{mmol}, 42 \%)$.

$1(\boldsymbol{R}):[\alpha]_{20}^{\mathrm{D}}=+4.1\left(c=1.04, \mathrm{CHCl}_{3}\right) .{ }^{1} \mathrm{H}$ NMR $\left(250 \mathrm{MHz}, \mathrm{CDCl}_{3}\right)$ : $\delta=5.83$ (ddd, $J=16.0,10.5,5.5 \mathrm{~Hz}, 1 \mathrm{H}, \mathrm{CH}=\mathrm{CHaHb}), 5.25$ (dt, $J=17.2$, $1.5 \mathrm{~Hz}, 1 \mathrm{H}, \mathrm{CH}=\mathrm{CHaHb}$ ), 5.09 (dt, $J=10.5,1.4 \mathrm{~Hz}, 1 \mathrm{H}, \mathrm{CH}=\mathrm{CHaHb}$ ), 4.45 (m, 1H, CHOH), 3.2 (br s, $1 \mathrm{H}, \mathrm{CHOH}), 2.47$ (dd, $J=16.1,4.7 \mathrm{~Hz}$, $1 \mathrm{H}, \mathrm{COCHaHb}), 2.38$ (dd, $J=16.1,7.7 \mathrm{~Hz}, 1 \mathrm{H}, \mathrm{COCHaHb}), 1.42$ (s, 9H, $t$ $\mathrm{Bu})$ ppm. ${ }^{13} \mathrm{C}$ NMR (62.5 MHz, $\left.\mathrm{CDCl}_{3}\right): \delta=171.7,139.0,115.2,81.4$, 69.1, 42.2, 28.1 ppm. IR (film): $\nu_{\max }=3434,2979,2931,1726,1645$, 1393, 1368, 1256, 1157, 1039, 993, 924, 842, $763 \mathrm{~cm}^{-1}$. HRMS (ESI): calcd for $\mathrm{C}_{9} \mathrm{H}_{16} \mathrm{O}_{3}[\mathrm{M}+\mathrm{Na}]^{+} 195.0997$ found 195.1002 .

$3(\boldsymbol{S}):[\alpha]_{20}^{\mathrm{D}}=-5.5\left(c=1.09, \mathrm{CHCl}_{3}\right) .{ }^{1} \mathrm{H}$ NMR $\left(250 \mathrm{MHz}, \mathrm{CDCl}_{3}\right)$ : $\delta=5.81$ (ddd, $J=17.0,10.5,6.2 \mathrm{~Hz}, 1 \mathrm{H}, \mathrm{CH}=\mathrm{CHaHb}), 5.59(\mathrm{~m}, 1 \mathrm{H}$, $\mathrm{CHOCOCH}_{3}$ ), 5.28 (dt, $\left.J=17.2,1.2 \mathrm{~Hz}, 1 \mathrm{H}, \mathrm{CH}=\mathrm{CHaHb}\right), 5.18$ (dt, $J=10.5,1.2 \mathrm{~Hz}, 1 \mathrm{H}, \mathrm{CH}=\mathrm{CHaHb}), 2.60$ (dd, $J=15.3,7.9 \mathrm{~Hz}, 1 \mathrm{H}, t \mathrm{BuO}-$ COCHaHb), 2.50 (dd, $J=15.3,5.9 \mathrm{~Hz}, 1 \mathrm{H}, t \mathrm{BuOCOCHaHb}), 2.04(\mathrm{~s}, 3 \mathrm{H}$, $\left.\mathrm{OCOCH}_{3}\right), 1.42(\mathrm{~s}, 9 \mathrm{H}, \mathrm{t}-\mathrm{Bu}) \mathrm{ppm} .{ }^{13} \mathrm{C} \mathrm{NMR}\left(62.5 \mathrm{MHz}, \mathrm{CDCl}_{3}\right)$ : $\delta=169.9,169.1,135.3,117.4,81.2,71.2,40.8,28.1,21.2$ ppm. IR (film): $\nu_{\max }=2979,2933,1736,1646,1457,1369,1290,1235,1159,1024$, 991, 947, 847, $764 \mathrm{~cm}^{-1}$. HRMS (ESI): calcd for $\mathrm{C}_{11} \mathrm{H}_{18} \mathrm{O}_{4}[\mathrm{M}+\mathrm{Na}]^{+}$ 237.1103 found 237.1109 .

\section{2. (S) $t$-Butyl-3-(((S)-3-acetoxy-pent-4-enoyl)oxy)-pent-4- enoate 4}

To a solution of alcohol $\mathbf{1}(\boldsymbol{R})(514 \mathrm{mg}, 2.98 \mathrm{mmol})$ in toluene (15 mL) were added $\mathrm{PPh}_{3}(1.46 \mathrm{~g}, 5.57 \mathrm{mmol}, 1.9$ equiv) and acid 2 (S) $(550 \mathrm{mg}, 3.48 \mathrm{mmol}, 1.2$ equiv) in toluene $(5 \mathrm{~mL})$ at room temperature. After $10 \mathrm{~min}$, diethyl azodicarboxylate $(0.90 \mathrm{~mL}, 1.0 \mathrm{~g}$, $5.75 \mathrm{mmol}, 1.9$ equiv) was added dropwise over a period of $15 \mathrm{~min}$. The mixture was stirred at room temperature for $1 \mathrm{~h}$ and quenched with $\mathrm{H}_{2} \mathrm{O}$ and $\mathrm{Et}_{2} \mathrm{O}$. The layers were separated and the aqueous layer was extracted with $\mathrm{Et}_{2} \mathrm{O}$. The combined extracts were dried over $\mathrm{MgSO}_{4}$ and concentrated to give a residue which was purified by silica gel column chromatography (EtOAc/Petroleum ether 1:9) to afford 4 (628 mg, $2.01 \mathrm{mmol}, 68 \%)$.

4: $[\alpha]_{20}^{\mathrm{D}}=-8.0\left(c=0.75, \mathrm{CHCl}_{3}\right) .{ }^{1} \mathrm{H}$ NMR $\left(250 \mathrm{MHz}, \mathrm{CDCl}_{3}\right)$ : $\delta=5.88-5.69(\mathrm{~m}, 2 \mathrm{H}, 2 \mathrm{CH}=\mathrm{CHaHb}), 5.66-5.54(\mathrm{~m}, 2 \mathrm{H}, 2 \mathrm{CH}-\mathrm{OCO})$, $5.32-5.14\left(\mathrm{~m}, 4 \mathrm{H}, 2 \mathrm{CH}=\mathrm{CH}_{2}\right), 2.72-2.44\left(\mathrm{~m}, 4 \mathrm{H}, 2 \mathrm{COCH}_{2}\right), 2.02(\mathrm{~s}$, 
$\left.3 \mathrm{H}, \mathrm{OCOCH}_{3}\right), 1.41$ (s, 9H, t-Bu) ppm. ${ }^{13} \mathrm{C} \mathrm{NMR} \mathrm{(62.5} \mathrm{MHz,} \mathrm{CDCl}_{3}$ ): $\delta=169.9,168.9,168.6,135.0,134.9,117.8,117.6,81.2,71.6,70.7,40.6$, 39.5, 28.1, $21.1 \mathrm{ppm}$. IR (film): $\nu_{\max }=3090,2979,2934,1739,1647$, $1456,1369,1236,1160,1025,938,844,764 \mathrm{~cm}^{-1}$. HRMS (ESI): calcd for $\mathrm{C}_{16} \mathrm{H}_{24} \mathrm{O}_{6}[\mathrm{M}+\mathrm{Na}]^{+} 335.1471$ found 335.1469.

4.2.1. General procedure for the cross-metathesis and hydrogenation, access to compound $\mathbf{6}(\mathrm{m}=2-17)$. To a solution of $\mathbf{3}(\boldsymbol{S})(1 \mathrm{mmol})$ in anhydrous ethyl ether $(10 \mathrm{~mL})$ were added Grubbs2 catalyst (42.4 mg, $50 \mu \mathrm{mol}, 5 \mathrm{~mol} \%$ ), CuI (13.3 mg, $70 \mu \mathrm{mol}, 7 \% \mathrm{eq})$ and alkene ( $4 \mathrm{mmol}, 4$ equiv) under an Ar atmosphere. The mixture was heated to $40{ }^{\circ} \mathrm{C}$ for $1 \mathrm{~h} 30$ until the color of the reaction mixture went from brown to dark green, corresponding to disappearance of starting material by tlc. After cooling to room temperature, the mixture was filtered through a short column of sand and $\mathrm{MgSO}_{4}$. The solid was washed with ethyl ether and the solvent was evaporated under reduced pressure. The obtained residue was used in the next step without further purification. To a solution of the residue in dry EtOAc $(10 \mathrm{~mL})$ at room temperature, a catalytic amount of $\mathrm{PtO}_{2}$ was added. The flask was purged and filled with $\mathrm{H}_{2}$ gas and the mixture was stirred vigorously at room temperature for $2 \mathrm{~h}$. The reaction was filtered through a Celite pad and washed with EtOAc. The solvent was removed and the crude product was purified by silica gel column chromatography (EtOAc/Petroleum ether, $1: 19)$ to afford the corresponding adduct 6 ( $m=2$ to $m=17$ ) (yield from 61 to $79 \%$ for two steps).

4.2.1.1. (R) t-Butyl 3-acetoxy-octanoate (6, $m=2$ ). Yield $64 \%$. $[\alpha]_{20}^{\mathrm{D}}=+3.9\left(c=1.75, \quad \mathrm{CHCl}_{3}\right) .{ }^{1} \mathrm{H} \quad \mathrm{NMR} \quad\left(250 \mathrm{MHz}, \mathrm{CDCl}_{3}\right)$ : $\delta=5.26-5.08(\mathrm{~m}, 1 \mathrm{H}, \mathrm{CH}-\mathrm{O}), 2.52-2.37\left(\mathrm{~m}, 2 \mathrm{H}, \mathrm{COCH}_{2}\right), 2.0(\mathrm{~s}, 3 \mathrm{H}$, $\left.\mathrm{COCH}_{3}\right), 1.66-1.48\left(\mathrm{~m}, 2 \mathrm{H}, \mathrm{CH}-\mathrm{CH}_{2}\right), 1.41(\mathrm{~s}, 9 \mathrm{H}, t-\mathrm{Bu}), 1.35-1.15(\mathrm{~m}$, $\left.6 \mathrm{H},\left(\mathrm{CH}_{2}\right)_{3} \mathrm{CH}_{3}\right), 0.85\left(\mathrm{t}, 3 \mathrm{H}, \mathrm{CH}_{3}\right)$ ppm. ${ }^{13} \mathrm{C} \mathrm{NMR}\left(62.9 \mathrm{MHz}, \mathrm{CDCl}_{3}\right)$ : $\delta=170.4,169.7,80.9,70.9,40.7,34.1,31.7,28.1,24.9,22.6,21.2$, $14.1 \mathrm{ppm}$. IR (film): $\nu_{\max }=2958,2933,2862,1743,1456,1369,1240$, $1159,1026,949,845 \mathrm{~cm}^{-1}$. HRMS (ESI+) $\mathrm{m} / z$ calcd for $\mathrm{C}_{14} \mathrm{H}_{26} \mathrm{O}_{4} \mathrm{Na}$ $[\mathrm{M}+\mathrm{Na}]^{+}$281.1729, found 281.1723 .

4.2.1.2. (R) t-Butyl 3-acetoxy-nonanoate (6, $m=3$ ). Yield $69 \%$. $[\alpha]_{20}^{\mathrm{D}}=+2.3 \quad\left(c=3.12, \quad \mathrm{CHCl}_{3}\right) .{ }^{1} \mathrm{H} \quad \mathrm{NMR} \quad\left(250 \mathrm{MHz}, \mathrm{CDCl}_{3}\right)$ : $\delta=5.24-5.09(\mathrm{~m}, 1 \mathrm{H}, \mathrm{CH}-\mathrm{O}), 2.49-2.34\left(\mathrm{~m}, 2 \mathrm{H}, \mathrm{COCH}_{2}\right), 1.97(\mathrm{~s}, 3 \mathrm{H}$, $\left.\mathrm{COCH}_{3}\right), 1.64-1.46\left(\mathrm{~m}, 2 \mathrm{H}, \mathrm{CH}-\mathrm{CH}_{2}\right), 1.38(\mathrm{~s}, 9 \mathrm{H}, t-\mathrm{Bu}), 1.33-1.12(\mathrm{~m}$, $\left.8 \mathrm{H},\left(\mathrm{CH}_{2}\right)_{4} \mathrm{CH}_{3}\right), 0.82\left(\mathrm{t}, 3 \mathrm{H}, \mathrm{CH}_{3}\right)$ ppm. ${ }^{13} \mathrm{C} \mathrm{NMR}\left(62.9 \mathrm{MHz}, \mathrm{CDCl}_{3}\right)$ : $\delta=170.3,169.7,80.8,70.9,40.7,34.1,31.7,29.1,28.0,25.1,22.6,21.1$, $14.1 \mathrm{ppm}$. IR (film): $\nu_{\max }=2959,2930,2860,1740,1461,1368,1240$, $1158,1028,951,847 \mathrm{~cm}^{-1}$. HRMS (ESI+) $\mathrm{m} / z$ calcd for $\mathrm{C}_{15} \mathrm{H}_{28} \mathrm{O}_{4} \mathrm{Na}$ $[\mathrm{M}+\mathrm{Na}]^{+}$295.1885, found 295.1891.

4.2.1.3. (R) t-Butyl 3-acetoxy-decanoate (6, $m=4)$. Yield $79 \%$. $[\alpha]_{20}^{\mathrm{D}}=+4.5 \quad\left(c=2.90, \mathrm{CHCl}_{3}\right) .{ }^{1} \mathrm{H} \quad \mathrm{NMR} \quad\left(250 \mathrm{MHz}, \mathrm{CDCl}_{3}\right)$ : $\delta=5.24-5.08(\mathrm{~m}, 1 \mathrm{H}, \mathrm{CH}-\mathrm{O}), 2.52-2.37\left(\mathrm{~m}, 2 \mathrm{H}, \mathrm{COCH}_{2}\right), 1.99(\mathrm{~s}, 3 \mathrm{H}$, $\left.\mathrm{COCH}_{3}\right), 1.67-1.48\left(\mathrm{~m}, 2 \mathrm{H}, \mathrm{CH}-\mathrm{CH}_{2}\right), 1.41(\mathrm{~s}, 9 \mathrm{H}, t-\mathrm{Bu}), 1.35-1.15(\mathrm{~m}$, $\left.10 \mathrm{H},\left(\mathrm{CH}_{2}\right)_{5} \mathrm{CH}_{3}\right), 0.86\left(\mathrm{t}, 3 \mathrm{H}, \mathrm{CH}_{3}\right) \mathrm{ppm} .{ }^{13} \mathrm{C} \mathrm{NMR}\left(62.9 \mathrm{MHz}, \mathrm{CDCl}_{3}\right)$ : $\delta=170.4,169.8,80.8,70.9,40.7,34.2,32.0,29.7,29.5,28.1,25.2,22.8$, 21.2, $14.2 \mathrm{ppm}$. IR (film): $\nu_{\max }=2959,2928,2858,1739,1461,1369$, 1240, 1158, 1027, 952, $846 \mathrm{~cm}^{-1}$. HRMS (ESI+) $\mathrm{m} / \mathrm{z}$ calcd for $\mathrm{C}_{16} \mathrm{H}_{30} \mathrm{O}_{4} \mathrm{Na}[\mathrm{M}+\mathrm{Na}]^{+}$309.2042, found 309.2047.

4.2.1.4. (R) t-Butyl 3-acetoxy-undecanoate (6, $m=5)$. Yield 76\%. $[\alpha]_{20}^{\mathrm{D}}=+3.7 \quad\left(c=3.50, \mathrm{CHCl}_{3}\right) .{ }^{1} \mathrm{H} \quad \mathrm{NMR} \quad\left(250 \mathrm{MHz}, \mathrm{CDCl}_{3}\right)$ : $\delta=5.24-5.07(\mathrm{~m}, 1 \mathrm{H}, \mathrm{CH}-\mathrm{O}), 2.51-2.36\left(\mathrm{~m}, 2 \mathrm{H}, \mathrm{COCH}_{2}\right), 2.00(\mathrm{~s}, 3 \mathrm{H}$, $\left.\mathrm{COCH}_{3}\right), 1.66-1.47\left(\mathrm{~m}, 2 \mathrm{H}, \mathrm{CH}-\mathrm{CH}_{2}\right), 1.41(\mathrm{~s}, 9 \mathrm{H}, t-\mathrm{Bu}), 1.33-1.15(\mathrm{~m}$, $\left.12 \mathrm{H},\left(\mathrm{CH}_{2}\right)_{6} \mathrm{CH}_{3}\right), 0.85\left(\mathrm{t}, 3 \mathrm{H}, \mathrm{CH}_{3}\right) \mathrm{ppm} .{ }^{13} \mathrm{C} \mathrm{NMR}\left(62.9 \mathrm{MHz}, \mathrm{CDCl}_{3}\right)$ : $\delta=170.4,169.8,80.9,70.9,40.7,34.2,31.9,29.5,29.4,29.3,28.1,25.2$, 22.7, 21.2, $14.2 \mathrm{ppm}$. IR (film): $\nu_{\max }=2956,2925,2854,1742,1458$, $1368,1238,1156,1024,950,845 \mathrm{~cm}^{-1}$. HRMS (ESI+) $\mathrm{m} / z$ calcd for $\mathrm{C}_{17} \mathrm{H}_{32} \mathrm{O}_{4} \mathrm{Na}[\mathrm{M}+\mathrm{Na}]^{+}$323.2198, found 323.2193.
4.2.1.5. (R) t-Butyl 3-acetoxy-dodecanoate $(\mathbf{6}, m=6)$. Yield $79 \%$. $[\alpha]_{20}^{\mathrm{D}}=+4.8 \quad\left(c=2.95, \mathrm{CHCl}_{3}\right) .{ }^{1} \mathrm{H} \quad \mathrm{NMR} \quad\left(250 \mathrm{MHz}, \mathrm{CDCl}_{3}\right)$ : $\delta=5.23-5.07(\mathrm{~m}, 1 \mathrm{H}, \mathrm{CH}-\mathrm{O}), 2.51-2.35\left(\mathrm{~m}, 2 \mathrm{H}, \mathrm{COCH}_{2}\right), 1.99(\mathrm{~s}, 3 \mathrm{H}$, $\left.\mathrm{COCH}_{3}\right), 1.65-1.46\left(\mathrm{~m}, 2 \mathrm{H}, \mathrm{CH}-\mathrm{CH}_{2}\right), 1.40(\mathrm{~s}, 9 \mathrm{H}, t-\mathrm{Bu}), 1.32-1.13(\mathrm{~m}$, $\left.14 \mathrm{H},\left(\mathrm{CH}_{2}\right)_{7} \mathrm{CH}_{3}\right), 0.84\left(\mathrm{t}, 3 \mathrm{H}, \mathrm{CH}_{3}\right) \mathrm{ppm} .{ }^{13} \mathrm{C} \mathrm{NMR}\left(62.9 \mathrm{MHz}, \mathrm{CDCl}_{3}\right)$ : $\delta=170.4,169.8,80.8,70.9,40.7,34.2,31.9,29.7,29.6,29.5,29.4,28.1$, 25.2, 22.7, 21.2, $14.2 \mathrm{ppm}$. IR (film): $\nu_{\max }=2951,2925,2854,1742$, $1459,1368,1239,1158,1025,952,847 \mathrm{~cm}^{-1}$. HRMS (ESI+) $\mathrm{m} / z$ calcd for $\mathrm{C}_{18} \mathrm{H}_{34} \mathrm{O}_{4} \mathrm{Na}[\mathrm{M}+\mathrm{Na}]^{+}$337.2355, found 337.2363.

4.2.1.6. (R) t-Butyl 3-acetoxy-tridecanoate (6, $m=7$ ). Yield $67 \%$. $[\alpha]_{20}^{\mathrm{D}}=+3.5 \quad\left(c=1.94, \mathrm{CHCl}_{3}\right) .{ }^{1} \mathrm{H} \quad \mathrm{NMR} \quad\left(250 \mathrm{MHz}, \mathrm{CDCl}_{3}\right)$ : $\delta=5.24-5.08(\mathrm{~m}, 1 \mathrm{H}, \mathrm{CH}-\mathrm{O}), 2.52-2.37\left(\mathrm{~m}, 2 \mathrm{H}, \mathrm{COCH}_{2}\right), 2.00(\mathrm{~s}, 3 \mathrm{H}$, $\left.\mathrm{COCH}_{3}\right), 1.66-1.47\left(\mathrm{~m}, 2 \mathrm{H}, \mathrm{CH}-\mathrm{CH}_{2}\right), 1.41(\mathrm{~s}, 9 \mathrm{H}, t-\mathrm{Bu}), 1.35-1.14(\mathrm{~m}$, $\left.16 \mathrm{H},\left(\mathrm{CH}_{2}\right)_{8} \mathrm{CH}_{3}\right), 0.85\left(\mathrm{t}, 3 \mathrm{H}, \mathrm{CH}_{3}\right) \mathrm{ppm} .{ }^{13} \mathrm{C} \mathrm{NMR}\left(62.9 \mathrm{MHz}, \mathrm{CDCl}_{3}\right)$ : $\delta=170.4,169.8,80.8,70.9,40.7,34.2,32.0,29.7,29.6,29.6,29.5,29.4$, 28.1, 25.2, 22.8, 21.2, 14.2 ppm. IR (film): $\nu_{\max }=2954,2926,2855$, $1742,1456,1368,1239,1156,1026,952,845 \mathrm{~cm}^{-1}$. HRMS (ESI+) $\mathrm{m} / \mathrm{z}$ calcd for $\mathrm{C}_{19} \mathrm{H}_{36} \mathrm{O}_{4} \mathrm{Na}[\mathrm{M}+\mathrm{Na}]^{+}$351.2511, found 351.2515 .

4.2.1.7. (R) t-Butyl 3-acetoxy-tetradecanoate (6, $m=8$ ). Yield 62\%. $[\alpha]_{20}^{\mathrm{D}}=+3.3\left(c=2.66, \mathrm{CHCl}_{3}\right) .{ }^{1} \mathrm{H}$ NMR $\left(250 \mathrm{MHz}, \mathrm{CDCl}_{3}\right)$ : $\delta=5.25-5.08(\mathrm{~m}, 1 \mathrm{H}, \mathrm{CH}-\mathrm{O}), 2.53-2.37\left(\mathrm{~m}, 2 \mathrm{H}, \mathrm{COCH}_{2}\right), 2.01(\mathrm{~s}, 3 \mathrm{H}$, $\left.\mathrm{COCH}_{3}\right), 1.67-1.48\left(\mathrm{~m}, 2 \mathrm{H}, \mathrm{CH}-\mathrm{CH}_{2}\right), 1.42(\mathrm{~s}, 9 \mathrm{H}, t-\mathrm{Bu}), 1.37-1.16(\mathrm{~m}$, $\left.18 \mathrm{H},\left(\mathrm{CH}_{2}\right)_{9} \mathrm{CH}_{3}\right), 0.86\left(\mathrm{t}, 3 \mathrm{H}, \mathrm{CH}_{3}\right)$ ppm. ${ }^{13} \mathrm{C} \mathrm{NMR}\left(62.9 \mathrm{MHz}, \mathrm{CDCl}_{3}\right)$ : $\delta=170.5,169.8,80.9,71.0,40.7,34.2,32.0,29.7,29.7,29.6,29.6,29.5$, 29.5, 28.1, 25.2, 22.8, 21.2, $14.2 \mathrm{ppm}$. IR (film): $\nu_{\max }=2958,2925$, 2855, 1743, 1461, 1368, 1240, 1156, 1025, 952, $847 \mathrm{~cm}^{-1}$. HRMS (ESI+) $m / z$ calcd for $\mathrm{C}_{20} \mathrm{H}_{38} \mathrm{O}_{4} \mathrm{Na}[\mathrm{M}+\mathrm{Na}]^{+} 365.2668$, found 365.2676 .

4.2.1.8. (R) t-Butyl 3-acetoxy-pentadecanoate $6(m=9)$. Yield 61\%. $[\alpha]_{20}^{\mathrm{D}}=+2.6\left(c=4.01, \mathrm{CHCl}_{3}\right) .{ }^{1} \mathrm{H}$ NMR $\left(250 \mathrm{MHz}, \mathrm{CDCl}_{3}\right)$ : $\delta=5.24-5.10(\mathrm{~m}, 1 \mathrm{H}, \mathrm{CH}-\mathrm{O}), 2.53-2.37\left(\mathrm{~m}, 2 \mathrm{H}, \mathrm{COCH}_{2}\right), 2.02(\mathrm{~s}, 3 \mathrm{H}$, $\left.\mathrm{COCH}_{3}\right), 1.67-1.49\left(\mathrm{~m}, 2 \mathrm{H}, \mathrm{CH}-\mathrm{CH}_{2}\right), 1.42(\mathrm{~s}, 9 \mathrm{H}, t-\mathrm{Bu}), 1.36-1.17(\mathrm{~m}$, $\left.20 \mathrm{H},\left(\mathrm{CH}_{2}\right){ }_{10} \mathrm{CH}_{3}\right), 0.86\left(\mathrm{t}, 3 \mathrm{H}, \mathrm{CH}_{3}\right) \mathrm{ppm} .{ }^{13} \mathrm{C} \mathrm{NMR}(62.9 \mathrm{MHz}$, $\left.\mathrm{CDCl}_{3}\right): \delta=170.5,169.9,80.9,71.0,40.7,34.2,32.0,29.8,29.8,29.8$, 29.7, 29.6, 29.5, 29.5, 28.1, 25.2, 22.8, 21.2, 14.2 ppm. IR (film): $\nu_{\max }=2958,2924,2854,1743,1467,1368,1239,1158,1026,953$, $845 \mathrm{~cm}^{-1}$. HRMS (ESI+) $\mathrm{m} / \mathrm{z}$ calcd for $\mathrm{C}_{21} \mathrm{H}_{40} \mathrm{O}_{4} \mathrm{Na}[\mathrm{M}+\mathrm{Na}]^{+}$ 379.2824, found 379.2813 .

4.2.1.9. (R) t-Butyl 3-acetoxy-hexadecanoate (6, $m=10)$. Yield 75\%. $[\alpha]_{20}^{\mathrm{D}}=+2.1 \quad\left(c=4.46, \mathrm{CHCl}_{3}\right) .{ }^{1} \mathrm{H} \mathrm{NMR}\left(250 \mathrm{MHz}, \mathrm{CDCl}_{3}\right)$ : $\delta=5.24-5.10(\mathrm{~m}, 1 \mathrm{H}, \mathrm{CH}-\mathrm{O}), 2.52-2.37\left(\mathrm{~m}, 2 \mathrm{H}, \mathrm{COCH}_{2}\right), 2.00(\mathrm{~s}, 3 \mathrm{H}$, $\left.\mathrm{COCH}_{3}\right), 1.66-1.48\left(\mathrm{~m}, 2 \mathrm{H}, \mathrm{CH}-\mathrm{CH}_{2}\right), 1.41(\mathrm{~s}, 9 \mathrm{H}, t-\mathrm{Bu}), 1.36-1.16(\mathrm{~m}$, $\left.22 \mathrm{H},\left(\mathrm{CH}_{2}\right){ }_{11} \mathrm{CH}_{3}\right), 0.85\left(\mathrm{t}, 3 \mathrm{H}, \mathrm{CH}_{3}\right)$ ppm. ${ }^{13} \mathrm{C}$ NMR $(62.9 \mathrm{MHz}$, $\left.\mathrm{CDCl}_{3}\right): \delta=170.4,169.8,80.9,70.9,40.7,34.2,32.0,29.8,29.8,29.8$, $29.8,29.6,29.6,29.5,29.5,28.1,25.2,22.8,21.2,14.2$ ppm. IR (film): $\nu_{\max }=2957,2924,2853,1743,1467,1368,1239,1155,1026,952$, $847 \mathrm{~cm}^{-1}$. HRMS (ESI+) $\mathrm{m} / \mathrm{z}$ calcd for $\mathrm{C}_{22} \mathrm{H}_{42} \mathrm{O}_{4} \mathrm{Na}[\mathrm{M}+\mathrm{Na}]^{+}$ 393.2981, found 393.2975.

4.2.1.10. (R) t-Butyl 3-acetoxy-heptadecanoate $(\mathbf{6}, m=11)$. Yield 65\%. $[\alpha]_{20}^{\mathrm{D}}=+1.5\left(c=2.64, \mathrm{CHCl}_{3}\right) .{ }^{1} \mathrm{H}$ NMR $\left(250 \mathrm{MHz}, \mathrm{CDCl}_{3}\right)$ : $\delta=5.22-5.13(\mathrm{~m}, 1 \mathrm{H}, \mathrm{CH}-\mathrm{O}), 2.50-2.40\left(\mathrm{~m}, 2 \mathrm{H}, \mathrm{COCH}_{2}\right), 2.01(\mathrm{~s}, 3 \mathrm{H}$, $\left.\mathrm{COCH}_{3}\right), 1.63-1.50\left(\mathrm{~m}, 2 \mathrm{H}, \mathrm{CH}-\mathrm{CH}_{2}\right), 1.42(\mathrm{~s}, 9 \mathrm{H}, t-\mathrm{Bu}), 1.33-1.20(\mathrm{~m}$, $\left.24 \mathrm{H},\left(\mathrm{CH}_{2}\right){ }_{12} \mathrm{CH}_{3}\right), 0.86\left(\mathrm{t}, 3 \mathrm{H}, \mathrm{CH}_{3}\right) \mathrm{ppm} .{ }^{13} \mathrm{C} \mathrm{NMR}(62.9 \mathrm{MHz}$, $\left.\mathrm{CDCl}_{3}\right): \delta=170.4,169.6,80.9,71.0,40.7,34.2,32.0,29.8,29.8,29.8$, 29.8, 29.8, 29.7, 29.6, 29.5, 29.5, 28.1, 25.2, 22.8, 21.2, 14.2 ppm. IR (film): $\nu_{\max }=2958,2925,2853,1743,1466,1368,1239,1157,1025$, $952,847 \mathrm{~cm}^{-1}$. HRMS (ESI+) $\mathrm{m} / z$ calcd for $\mathrm{C}_{23} \mathrm{H}_{44} \mathrm{O}_{4} \mathrm{Na}[\mathrm{M}+\mathrm{Na}]^{+}$ 407.3137, found 407.3141.

4.2.1.11. (R) t-Butyl 3-acetoxy-octadecanoate (6, $m=12$ ). Yield 63\%. $[\alpha]_{20}^{\mathrm{D}}=+2.2\left(c=2.57, \mathrm{CHCl}_{3}\right) .{ }^{1} \mathrm{H}$ NMR $\left(250 \mathrm{MHz}, \mathrm{CDCl}_{3}\right)$ : 
$\delta=5.22-5.13(\mathrm{~m}, 1 \mathrm{H}, \mathrm{CH}-\mathrm{O}), 2.50-2.41\left(\mathrm{~m}, 2 \mathrm{H}, \mathrm{COCH}_{2}\right), 2.01(\mathrm{~s}, 3 \mathrm{H}$, $\left.\mathrm{COCH}_{3}\right), 1.63-1.50\left(\mathrm{~m}, 2 \mathrm{H}, \mathrm{CH}-\mathrm{CH}_{2}\right), 1.42(\mathrm{~s}, 9 \mathrm{H}, t-\mathrm{Bu}), 1.33-1.18(\mathrm{~m}$, $\left.26 \mathrm{H},\left(\mathrm{CH}_{2}\right){ }_{13} \mathrm{CH}_{3}\right), 0.86\left(\mathrm{t}, 3 \mathrm{H}, \mathrm{CH}_{3}\right) \mathrm{ppm} .{ }^{13} \mathrm{C} \mathrm{NMR}(62.9 \mathrm{MHz}$, $\left.\mathrm{CDCl}_{3}\right): \delta=170.4,169.8,80.9,71.0,40.7,34.2,32.1,29.8,29.8,29.8$, $29.8,29.8,29.8,29.7,29.6,29.5,29.5,28.1,25.2,22.8,21.2,14.2 \mathrm{ppm}$. IR (film): $\nu_{\max }=2958,2925,2853,1743,1465,1368,1239,1157,1025$, $951,847 \mathrm{~cm}^{-1}$. HRMS (ESI+) $\mathrm{m} / z$ calcd for $\mathrm{C}_{24} \mathrm{H}_{46} \mathrm{O}_{4} \mathrm{Na}[\mathrm{M}+\mathrm{Na}]^{+}$ 421.3294, found 421.3291 .

4.2.1.12. (R) t-Butyl 3-acetoxy-nonadecanoate (6, $m=13)$. Yield 76\%. $[\alpha]_{20}^{\mathrm{D}}=+2.0\left(c=2.05, \mathrm{CHCl}_{3}\right) .{ }^{1} \mathrm{H}$ NMR $\left(250 \mathrm{MHz}, \mathrm{CDCl}_{3}\right)$ : $\delta=5.23-5.13(\mathrm{~m}, 1 \mathrm{H}, \mathrm{CH}-\mathrm{O}), 2.53-2.38\left(\mathrm{~m}, 2 \mathrm{H}, \mathrm{COCH}_{2}\right), 2.02(\mathrm{~s}, 3 \mathrm{H}$, $\left.\mathrm{COCH}_{3}\right), 1.64-1.49\left(\mathrm{~m}, 2 \mathrm{H}, \mathrm{CH}-\mathrm{CH}_{2}\right), 1.42(\mathrm{~s}, 9 \mathrm{H}, t-\mathrm{Bu}), 1.35-1.18(\mathrm{~m}$, $\left.28 \mathrm{H},\left(\mathrm{CH}_{2}\right){ }_{14} \mathrm{CH}_{3}\right), 0.87\left(\mathrm{t}, 3 \mathrm{H}, \mathrm{CH}_{3}\right) \mathrm{ppm} .{ }^{13} \mathrm{C} \mathrm{NMR}(62.9 \mathrm{MHz}$, $\left.\mathrm{CDCl}_{3}\right): \delta=170.5,169.9,80.9,71.0,40.7,34.2,32.1,29.8,29.8,29.8$, 29.8, 29.8, 29.8, 29.8, 29.7, 29.6, 29.5, 29.5, 28.1, 25.3, 22.8, 21.3, 14.3 ppm. IR (film): $\nu_{\max }=2957,2923,2853,1743,1467,1368,1239$, $1158,1025,953,847 \mathrm{~cm}^{-1}$. HRMS (ESI+) $\mathrm{m} / z$ calcd for $\mathrm{C}_{25} \mathrm{H}_{48} \mathrm{O}_{4} \mathrm{Na}$ $[\mathrm{M}+\mathrm{Na}]^{+}$435.3450, found 435.3445 .

4.2.1.13. (R) t-Butyl 3-acetoxy-icosanoate (6, $m=14)$. Yield $62 \%$. $[\alpha]_{20}^{\mathrm{D}}=+1.2 \quad\left(c=2.22, \mathrm{CHCl}_{3}\right) .{ }^{1} \mathrm{H} \quad \mathrm{NMR} \quad\left(250 \mathrm{MHz}, \mathrm{CDCl}_{3}\right)$ : $\delta=5.23-5.11(\mathrm{~m}, 1 \mathrm{H}, \mathrm{CH}-\mathrm{O}), 2.53-2.38\left(\mathrm{~m}, 2 \mathrm{H}, \mathrm{COCH}_{2}\right), 2.02(\mathrm{~s}, 3 \mathrm{H}$, $\left.\mathrm{COCH}_{3}\right), 1.65-1.49\left(\mathrm{~m}, 2 \mathrm{H}, \mathrm{CH}-\mathrm{CH}_{2}\right), 1.42(\mathrm{~s}, 9 \mathrm{H}, t-\mathrm{Bu}), 1.37-1.17(\mathrm{~m}$, $\left.30 \mathrm{H},\left(\mathrm{CH}_{2}\right){ }_{15} \mathrm{CH}_{3}\right), 0.87\left(\mathrm{t}, 3 \mathrm{H}, \mathrm{CH}_{3}\right)$ ppm. ${ }^{13} \mathrm{C} \mathrm{NMR}(62.9 \mathrm{MHz}$, $\left.\mathrm{CDCl}_{3}\right): \delta=170.5,169.9,80.9,70.9,40.7,34.2,32.1,29.8,29.8,29.8$, 29.8, 29.8, 29.8, 29.8, 29.8, 29.7, 29.6, 29.5, 29.5, 28.1, 25.3, 22.8, 21.2, 14.2 ppm. IR (film): $\nu_{\max }=2957,2924,2853,1744,1464,1368$, 1239, 1158, 1026, 952, $845 \mathrm{~cm}^{-1}$. HRMS (ESI+) $\mathrm{m} / z$ calcd for $\mathrm{C}_{26} \mathrm{H}_{50} \mathrm{O}_{4} \mathrm{Na}[\mathrm{M}+\mathrm{Na}]^{+} 449.3607$, found 449.3612 .

4.2.1.14. (R) t-Butyl 3-acetoxy-eicosanoate $(6, m=15)$. Yield $68 \%$. $[\alpha]_{20}^{\mathrm{D}}=+1.6\left(c=2.14, \mathrm{CHCl}_{3}\right) .{ }^{1} \mathrm{H} \mathrm{NMR}\left(250 \mathrm{MHz}, \mathrm{CDCl}_{3}\right): \delta=5.24-5.11$ $(\mathrm{m}, 1 \mathrm{H}, \mathrm{CH}-\mathrm{O}), 2.53-2.38\left(\mathrm{~m}, 2 \mathrm{H}, \mathrm{COCH}_{2}\right), 2.01\left(\mathrm{~s}, 3 \mathrm{H}, \mathrm{COCH}_{3}\right)$, 1.64-1.49 (m, 2H, CH- $\left.\mathrm{CH}_{2}\right), 1.42(\mathrm{~s}, 9 \mathrm{H}, t-\mathrm{Bu}), 1.36-1.17(\mathrm{~m}, 32 \mathrm{H}$, $\left.\left(\mathrm{CH}_{2}\right)_{16} \mathrm{CH}_{3}\right), 0.86\left(\mathrm{t}, 3 \mathrm{H}, \mathrm{CH}_{3}\right) \mathrm{ppm} .{ }^{13} \mathrm{C} \mathrm{NMR}\left(62.9 \mathrm{MHz}, \mathrm{CDCl}_{3}\right)$ : $\delta=170.4,169.8,80.9,71.0,40.7,34.2,32.1,29.8,29.8,29.8,29.8,29.8$, 29.8, 29.8, 29.8, 29.8, 29.7, 29.6, 29.5, 29.5, 28.1, 25.3, 22.8, 21.2, $14.2 \mathrm{ppm}$. IR (film): $\nu_{\max }=2955,2924,2853,1744,1466,1368,1239$, $1157,1025,952,847 \mathrm{~cm}^{-1}$. HRMS (ESI+) $\mathrm{m} / z$ calcd for $\mathrm{C}_{27} \mathrm{H}_{52} \mathrm{O}_{4} \mathrm{Na}$ $[\mathrm{M}+\mathrm{Na}]^{+}$463.3763, found 463.3755 .

4.2.1.15. (R) t-Butyl 3-acetoxy-docosanoate $(\mathbf{6}, m=16)$. Yield $64 \%$. $[\alpha]_{20}^{\mathrm{D}}=+2.7\left(c=2.0, \mathrm{CHCl}_{3}\right) .{ }^{1} \mathrm{H} \mathrm{NMR}\left(250 \mathrm{MHz}, \mathrm{CDCl}_{3}\right): \delta=5.25-5.11$ $(\mathrm{m}, 1 \mathrm{H}, \mathrm{CH}-\mathrm{O}), 2.54-2.38\left(\mathrm{~m}, 2 \mathrm{H}, \mathrm{COCH}_{2}\right), 2.02\left(\mathrm{~s}, 3 \mathrm{H}, \mathrm{COCH}_{3}\right)$, 1.64-1.49 (m, 2H, CH- $\left.\mathrm{CH}_{2}\right), 1.43(\mathrm{~s}, 9 \mathrm{H}, t-\mathrm{Bu}), 1.36-1.17(\mathrm{~m}, 34 \mathrm{H}$, $\left.\left(\mathrm{CH}_{2}\right)_{17} \mathrm{CH}_{3}\right), 0.87$ (t, 3H, CH 3$)$ ppm. ${ }^{13} \mathrm{C}$ NMR $\left(62.9 \mathrm{MHz}, \mathrm{CDCl}_{3}\right)$ : $\delta=170.5,169.9,80.9,71.0,40.8,34.2,32.1,29.8,29.8,29.8,29.8,29.8$, $29.8,29.8,29.8,29.8,29.8,29.7,29.6,29.6,29.5,28.2,25.3,22.8$, $21.3,14.3$ ppm. IR (film): $\nu_{\max }=2954,2922,2852,1740,1467,1368$, $1240,1158,1026,952,849 \mathrm{~cm}^{-1}$. HRMS (ESI+) $\mathrm{m} / z$ calcd for $\mathrm{C}_{28} \mathrm{H}_{54} \mathrm{O}_{4} \mathrm{Na}[\mathrm{M}+\mathrm{Na}]^{+}$477.3920, found 477.3930 .

4.2.1.16. (R) t-Butyl 3-acetoxy-tricosanoate (6, $m=17$ ). Yield $68 \%$. $[\alpha]_{20}^{\mathrm{D}}=+1.1 \quad\left(c=3.80, \mathrm{CHCl}_{3}\right) .{ }^{1} \mathrm{H} \quad \mathrm{NMR} \quad\left(250 \mathrm{MHz}, \mathrm{CDCl}_{3}\right)$ : $\delta=5.24-5.11(\mathrm{~m}, 1 \mathrm{H}, \mathrm{CH}-\mathrm{O}), 2.53-2.38\left(\mathrm{~m}, 2 \mathrm{H}, \mathrm{COCH}_{2}\right), 2.02(\mathrm{~s}, 3 \mathrm{H}$, $\left.\mathrm{COCH}_{3}\right), 1.64-1.49\left(\mathrm{~m}, 2 \mathrm{H}, \mathrm{CH}-\mathrm{CH}_{2}\right), 1.42(\mathrm{~s}, 9 \mathrm{H}, t-\mathrm{Bu}), 1.37-1.17(\mathrm{~m}$, $\left.36 \mathrm{H},\left(\mathrm{CH}_{2}\right){ }_{18} \mathrm{CH}_{3}\right), 0.87\left(\mathrm{t}, 3 \mathrm{H}, \mathrm{CH}_{3}\right) \mathrm{ppm} .{ }^{13} \mathrm{C} \mathrm{NMR}(62.9 \mathrm{MHz}$, $\left.\mathrm{CDCl}_{3}\right): \delta=170.4,169.8,80.9,71.0,40.7,34.2,32.1,29.8,29.8,29.8$, 29.8, 29.8, 29.8, 29.8, 29.8, 29.8, 29.8, 29.8, 29.7, 29.6, 29.5, 29.5, 28.1, 25.3, 22.8, 21.2, 14.3 ppm. IR (film): $\nu_{\max }=2952,2921,2851$, $1736,1467,1368,1240,1158,1027,954,849 \mathrm{~cm}^{-1}$. HRMS (ESI+) $\mathrm{m} / z$ calcd for $\mathrm{C}_{29} \mathrm{H}_{56} \mathrm{O}_{4} \mathrm{Na}[\mathrm{M}+\mathrm{Na}]^{+} 491.4076$, found 491.4081 .

4.2.2. General deprotection procedure, access to compounds 7 ( $m=2$ to $m=17)$. Trifluoroacetic acid $(1 \mathrm{~mL})\left(20 \%\right.$ in $\left.\mathrm{CH}_{2} \mathrm{Cl}_{2}\right)$ was added to a solution of $\mathbf{6}$ ( $m=2$ to $m=17)(0.1 \mathrm{mmol})$ in dry $\mathrm{CH}_{2} \mathrm{Cl}_{2}(4 \mathrm{~mL})$. The reaction mixture was stirred at room temperature for $45 \mathrm{~min}$ before it was quenched with $\mathrm{H}_{2} \mathrm{O}(1 \mathrm{~mL})$. The layers were separated and the aqueous layer was extracted with $\mathrm{CH}_{2} \mathrm{Cl}_{2}(10 \mathrm{~mL})$. The combined organic layers were collected, washed with brine and dried over $\mathrm{MgSO}_{4}$. The solvent was concentrated under reduced pressure and filtered through a short column of $\mathrm{SiO}_{2}$ (EtOAc/Petroleum ether, $1: 4)$ to afford the corresponding acid $7(m=2$ to $m=17)$ (84-95\% yield).

4.2.2.1. (R) 3-Acetoxy-octanoic acid (7, $m=2$ ). Yield $84 \%$. $[\alpha]_{20}^{\mathrm{D}}=+1.8\left(c=0.75, \mathrm{CHCl}_{3}\right) .{ }^{1} \mathrm{H} \mathrm{NMR}\left(250 \mathrm{MHz}, \mathrm{CDCl}_{3}\right): \delta=9.72(\mathrm{br}$ $\mathrm{s}, \mathrm{COOH}), 5.26-5.09(\mathrm{~m}, 1 \mathrm{H}, \mathrm{CH}-\mathrm{O}), 2.68-2.51\left(\mathrm{~m}, 2 \mathrm{H}, \mathrm{COCH}_{2}\right), 2.03$ $\left(\mathrm{s}, 3 \mathrm{H}, \mathrm{COCH}_{3}\right), 1.71-1.50\left(\mathrm{~m}, 2 \mathrm{H}, \mathrm{CH}-\mathrm{CH}_{2}\right), 1.40-1.19(\mathrm{~m}, 6 \mathrm{H}$, $\left.\left(\mathrm{CH}_{2}\right)_{3} \mathrm{CH}_{3}\right), 0.86\left(\mathrm{t}, 3 \mathrm{H}, \mathrm{CH}_{3}\right)$ ppm. $\left.{ }^{13} \mathrm{C} \mathrm{NMR} \mathrm{(62.9} \mathrm{MHz}, \mathrm{CDCl}_{3}\right)$ : $\delta=176.7,171.1,70.6,39.0,34.0,31.6,24.9,22.6,21.2,14.0 \mathrm{ppm}$. IR (film): $\nu_{\max }=3305,3021,2933,2862,1743,1456,1369,1240,1159$, 1026, $757 \mathrm{~cm}^{-1}$. HRMS (ESI+) $\mathrm{m} / z$ calcd for $\mathrm{C}_{10} \mathrm{H}_{18} \mathrm{O}_{4} \mathrm{Na}[\mathrm{M}+\mathrm{Na}]^{+}$ 225.1103, found 225.1107.

4.2.2.2. (R) 3-Acetoxy-nonanoic acid (7, $m=3$ ). Yield 86\%. $[\alpha]_{20}^{\mathrm{D}}=-3.1\left(c=0.75, \mathrm{CHCl}_{3}\right) .{ }^{1} \mathrm{H}$ NMR $\left(250 \mathrm{MHz}, \mathrm{CDCl}_{3}\right): \delta=6.71(\mathrm{br}$ $\mathrm{s}, \mathrm{COOH}), 5.27-5.13(\mathrm{~m}, 1 \mathrm{H}, \mathrm{CH}-\mathrm{O}), 2.69-2.52\left(\mathrm{~m}, 2 \mathrm{H}, \mathrm{COCH}_{2}\right), 2.04$ $\left(\mathrm{s}, 3 \mathrm{H}, \mathrm{COCH}_{3}\right), 1.72-1.53\left(\mathrm{~m}, 2 \mathrm{H}, \mathrm{CH}-\mathrm{CH}_{2}\right), 1.40-1.18(\mathrm{~m}, 8 \mathrm{H}$ $\left.\left(\mathrm{CH}_{2}\right)_{4} \mathrm{CH}_{3}\right), 0.87$ (t, 3H, CH$)$ ppm. $\left.{ }^{13} \mathrm{C} \mathrm{NMR} \mathrm{(62.9} \mathrm{MHz}, \mathrm{CDCl}_{3}\right)$ : $\delta=176.1,170.7,70.5,39.0,34.1,31.8,29.1,25.2,22.7,21.2,14.2 \mathrm{ppm}$. IR (film): $\nu_{\max }=3315,3015,2929,2859,1743,1715,1434,1376,1240$ $1029,758 \mathrm{~cm}^{-1}$. HRMS (ESI+) $\mathrm{m} / z$ calcd for $\mathrm{C}_{11} \mathrm{H}_{20} \mathrm{O}_{4} \mathrm{Na}[\mathrm{M}+\mathrm{Na}]^{+}$ 239.1259, found 239.1253 .

4.2.2.3. (R) 3-Acetoxy-decanoic acid (7, $m=4$ ). Yield $89 \%$. $[\alpha]_{20}^{\mathrm{D}}=-2.8\left(c=0.9, \mathrm{CHCl}_{3}\right) .{ }^{1} \mathrm{H} \mathrm{NMR}\left(250 \mathrm{MHz}, \mathrm{CDCl}_{3}\right): \delta=6.45$ (br s, $\mathrm{COOH}), 5.27-5.13(\mathrm{~m}, 1 \mathrm{H}, \mathrm{CH}-\mathrm{O}), 2.69-2.52\left(\mathrm{~m}, 2 \mathrm{H}, \mathrm{COCH}_{2}\right), 2.04$ $\left(\mathrm{s}, 3 \mathrm{H}, \mathrm{COCH}_{3}\right), 1.71-1.53\left(\mathrm{~m}, 2 \mathrm{H}, \mathrm{CH}-\mathrm{CH}_{2}\right), 1.40-1.18(\mathrm{~m}, 10 \mathrm{H}$, $\left.\left(\mathrm{CH}_{2}\right)_{5} \mathrm{CH}_{3}\right), 0.87\left(\mathrm{t}, 3 \mathrm{H}, \mathrm{CH}_{3}\right)$ ppm. ${ }^{13} \mathrm{C} \mathrm{NMR}\left(62.9 \mathrm{MHz}, \mathrm{CDCl}_{3}\right)$ : $\delta=176.1,170.7,70.5,38.9,34.1,31.9,29.4,29.3,25.3,22.8,21.2$, $14.2 \mathrm{ppm}$. IR (film): $\nu_{\max }=3320,3022,2928,2857,1738,1717,1434$, $1376,1245,1029,757 \mathrm{~cm}^{-1}$. HRMS (ESI+) $\mathrm{m} / z$ calcd for $\mathrm{C}_{12} \mathrm{H}_{22} \mathrm{O}_{4} \mathrm{Na}$ $[\mathrm{M}+\mathrm{Na}]^{+}$253.1416, found 253.1421.

4.2.2.4. (R) 3-Acetoxy-undecanoic acid (7, $m=5$ ). Yield $91 \%$ $[\alpha]_{20}^{\mathrm{D}}=-2.2\left(\mathrm{c}=1.0, \mathrm{CHCl}_{3}\right) .{ }^{1} \mathrm{H}$ NMR $\left(250 \mathrm{MHz}, \mathrm{CDCl}_{3}\right): \delta=6.82(\mathrm{br} \mathrm{s}$, $\mathrm{COOH}), 5.27-5.13(\mathrm{~m}, 1 \mathrm{H}, \mathrm{CH}-\mathrm{O}), 2.69-2.52\left(\mathrm{~m}, 2 \mathrm{H}, \mathrm{COCH}_{2}\right), 2.04$ $\left(\mathrm{s}, 3 \mathrm{H}, \mathrm{COCH}_{3}\right), 1.70-1.52\left(\mathrm{~m}, 2 \mathrm{H}, \mathrm{CH}-\mathrm{CH}_{2}\right), 1.41-1.17(\mathrm{~m}, 12 \mathrm{H}$, $\left.\left(\mathrm{CH}_{2}\right)_{6} \mathrm{CH}_{3}\right), 0.87\left(\mathrm{t}, 3 \mathrm{H}, \mathrm{CH}_{3}\right) \mathrm{ppm} .{ }^{13} \mathrm{C} \mathrm{NMR}\left(62.9 \mathrm{MHz}, \mathrm{CDCl}_{3}\right)$ : $\delta=176.2,170.7,70.5,39.0,34.1,32.0,29.5,29.5,29.3,25.2,22.8,21.2$, $14.2 \mathrm{ppm}$. IR (film): $\nu_{\max }=3331,3012,2926,2856,1743,1716,1433$, $1377,1239,1029,770 \mathrm{~cm}^{-1}$. HRMS (ESI+) $\mathrm{m} / z$ calcd for $\mathrm{C}_{13} \mathrm{H}_{24} \mathrm{O}_{4} \mathrm{Na}$ $[\mathrm{M}+\mathrm{Na}]^{+}$267.1572, found 267.1568 .

4.2.2.5. (R) 3-Acetoxy-dodecanoic acid (7, $m=6$ ). Yield $90 \%$ $[\alpha]_{20}^{\mathrm{D}}=-1.8\left(c=1.15, \mathrm{CHCl}_{3}\right) .{ }^{1} \mathrm{H} \mathrm{NMR}\left(250 \mathrm{MHz}, \mathrm{CDCl}_{3}\right): \delta=6.26$ (br s, $\mathrm{COOH}), 5.26-5.11(\mathrm{~m}, 1 \mathrm{H}, \mathrm{CH}-\mathrm{O}), 2.69-2.52\left(\mathrm{~m}, 2 \mathrm{H}, \mathrm{COCH}_{2}\right), 2.04$ $\left(\mathrm{s}, 3 \mathrm{H}, \mathrm{COCH}_{3}\right), 1.72-1.53\left(\mathrm{~m}, 2 \mathrm{H}, \mathrm{CH}-\mathrm{CH}_{2}\right), 1.40-1.17(\mathrm{~m}, 14 \mathrm{H}$, $\left.\left(\mathrm{CH}_{2}\right)_{7} \mathrm{CH}_{3}\right), 0.87\left(\mathrm{t}, 3 \mathrm{H}, \mathrm{CH}_{3}\right) \mathrm{ppm} .{ }^{13} \mathrm{C} \mathrm{NMR}\left(62.9 \mathrm{MHz}, \mathrm{CDCl}_{3}\right)$ : $\delta=176.0,170.7,70.5,38.9,34.1,32.0,29.6,29.6,29.5,29.4,25.3,22.8$, 21.2, $14.2 \mathrm{ppm}$. IR (film): $\nu_{\max }=3392,3028,2926,2855,1741,1717$, 1434, 1376, 1240, 1028, $757 \mathrm{~cm}^{-1}$. HRMS (ESI+) $\mathrm{m} / z$ calcd for $\mathrm{C}_{14} \mathrm{H}_{26} \mathrm{O}_{4} \mathrm{Na}[\mathrm{M}+\mathrm{Na}]^{+}$281.1729, found 281.1733.

4.2.2.6. (R) 3-Acetoxy-tridecanoic acid (7, $m=7$ ). Yield $93 \%$. $[\alpha]_{20}^{\mathrm{D}}=-2.5\left(c=0.75, \mathrm{CHCl}_{3}\right) .{ }^{1} \mathrm{H} \mathrm{NMR}\left(250 \mathrm{MHz}, \mathrm{CDCl}_{3}\right): \delta=7.15$ (br s, $\mathrm{COOH}), 5.26-5.12(\mathrm{~m}, 1 \mathrm{H}, \mathrm{CH}-\mathrm{O}), 2.69-2.52\left(\mathrm{~m}, 2 \mathrm{H}, \mathrm{COCH}_{2}\right), 2.04$ $\left(\mathrm{s}, 3 \mathrm{H}, \mathrm{COCH}_{3}\right), 1.71-1.52\left(\mathrm{~m}, 2 \mathrm{H}, \mathrm{CH}-\mathrm{CH}_{2}\right), 1.40-1.18(\mathrm{~m}, 16 \mathrm{H}$, $\left.\left(\mathrm{CH}_{2}\right)_{8} \mathrm{CH}_{3}\right), 0.87\left(\mathrm{t}, 3 \mathrm{H}, \mathrm{CH}_{3}\right) \mathrm{ppm} .{ }^{13} \mathrm{C} \mathrm{NMR}\left(62.9 \mathrm{MHz}, \mathrm{CDCl}_{3}\right)$ : $\delta=176.2,170.7,70.5,39.0,34.1,32.0,29.8,29.7,29.7,29.6,29.5,25.2$, 
22.8, 21.2, 14.2 ppm. IR (film): $\nu_{\max }=3315,3023,2926,2854,1736$, $1718,1466,1376,1241,1028,758 \mathrm{~cm}^{-1}$. HRMS (ESI+) $\mathrm{m} / z$ calcd for $\mathrm{C}_{15} \mathrm{H}_{28} \mathrm{O}_{4} \mathrm{Na}[\mathrm{M}+\mathrm{Na}]^{+}$295.1885, found 295.1879.

4.2.2.7. (R) 3-Acetoxy-tetradecanoic acid (7, $m=8$ ). Yield $94 \%$. $[\alpha]_{20}^{\mathrm{D}}=-2.2\left(c=1.1, \mathrm{CHCl}_{3}\right) .{ }^{1} \mathrm{H}$ NMR $\left(250 \mathrm{MHz}, \mathrm{CDCl}_{3}\right): \delta=6.25(\mathrm{br}$ $\mathrm{s}, \mathrm{COOH}), 5.27-5.12(\mathrm{~m}, 1 \mathrm{H}, \mathrm{CH}-\mathrm{O}), 2.69-2.52\left(\mathrm{~m}, 2 \mathrm{H}, \mathrm{COCH}_{2}\right)$, $2.04\left(\mathrm{~s}, 3 \mathrm{H}, \mathrm{COCH}_{3}\right), 1.71-1.52\left(\mathrm{~m}, 2 \mathrm{H}, \mathrm{CH}-\mathrm{CH}_{2}\right), 1.40-1.17(\mathrm{~m}$, $\left.18 \mathrm{H},\left(\mathrm{CH}_{2}\right)_{9} \mathrm{CH}_{3}\right), 0.87\left(\mathrm{t}, 3 \mathrm{H}, \mathrm{CH}_{3}\right) \mathrm{ppm} .{ }^{13} \mathrm{C} \mathrm{NMR}(62.9 \mathrm{MHz}$, $\left.\mathrm{CDCl}_{3}\right): \delta=176.0,170.7,70.5,39.0,34.1,32.1,29.8,29.8,29.7,29.6$, $29.5,29.5,25.2,22.8,21.2,14.2 \mathrm{ppm}$. IR (film): $\nu_{\max }=3379,3015$, 2925, 2854, 1739, 1716, 1466, 1375, 1241, 1028, $758 \mathrm{~cm}^{-1}$. HRMS $(\mathrm{ESI}+) \mathrm{m} / \mathrm{z}$ calcd for $\mathrm{C}_{16} \mathrm{H}_{30} \mathrm{O}_{4} \mathrm{Na}[\mathrm{M}+\mathrm{Na}]^{+}$309.2042, found 309.2033.

4.2.2.8. (R) 3-Acetoxy-pentadecanoic acid (7, $m=9$ ). Yield $95 \%$. $[\alpha]_{20}^{\mathrm{D}}=-2.5\left(c=1.4, \mathrm{CHCl}_{3}\right) .{ }^{1} \mathrm{H}$ NMR $\left(250 \mathrm{MHz}, \mathrm{CDCl}_{3}\right): \delta=7.31$ (br s, $\mathrm{COOH}), 5.27-5.12(\mathrm{~m}, 1 \mathrm{H}, \mathrm{CH}-\mathrm{O}), 2.69-2.52\left(\mathrm{~m}, 2 \mathrm{H}, \mathrm{COCH}_{2}\right), 2.04$ $\left(\mathrm{s}, 3 \mathrm{H}, \mathrm{COCH}_{3}\right), 1.72-1.52\left(\mathrm{~m}, 2 \mathrm{H}, \mathrm{CH}-\mathrm{CH}_{2}\right), 1.41-1.15(\mathrm{~m}, 20 \mathrm{H}$, $\left.\left(\mathrm{CH}_{2}\right)_{10} \mathrm{CH}_{3}\right), 0.87\left(\mathrm{t}, 3 \mathrm{H}, \mathrm{CH}_{3}\right)$ ppm. ${ }^{13} \mathrm{C}$ NMR $\left(62.9 \mathrm{MHz}, \mathrm{CDCl}_{3}\right)$ : $\delta=176.3,170.8,70.5,39.0,34.1,32.1,29.8,29.8,29.8,29.7,29.6,29.5$, 29.5, 25.2, 22.8, 21.2, $14.2 \mathrm{ppm}$. IR (film): $\nu_{\max }=3330,3028,2916$, $2849,1734,1715,1463,1376,1240,1037,759 \mathrm{~cm}^{-1}$. HRMS (ESI+) $\mathrm{m} / z$ calcd for $\mathrm{C}_{17} \mathrm{H}_{32} \mathrm{O}_{4} \mathrm{Na}[\mathrm{M}+\mathrm{Na}]^{+}$323.2198, found 323.2203.

4.2.2.9. (R) 3-Acetoxy-hexadecanoic acid (7, $m=10)$. Yield $94 \%$. $[\alpha]_{20}^{\mathrm{D}}=-2.1\left(c=1.3, \mathrm{CHCl}_{3}\right) .{ }^{1} \mathrm{H}$ NMR $\left(250 \mathrm{MHz}, \mathrm{CDCl}_{3}\right): \delta=7.16$ (br s, $\mathrm{COOH}), 5.27-5.13(\mathrm{~m}, 1 \mathrm{H}, \mathrm{CH}-\mathrm{O}), 2.69-2.52\left(\mathrm{~m}, 2 \mathrm{H}, \mathrm{COCH}_{2}\right), 2.04$ $\left(\mathrm{s}, 3 \mathrm{H}, \mathrm{COCH}_{3}\right), 1.71-1.53\left(\mathrm{~m}, 2 \mathrm{H}, \mathrm{CH}-\mathrm{CH}_{2}\right), 1.41-1.16(\mathrm{~m}, 22 \mathrm{H}$, $\left.\left(\mathrm{CH}_{2}\right)_{11} \mathrm{CH}_{3}\right), 0.88\left(\mathrm{t}, 3 \mathrm{H}, \mathrm{CH}_{3}\right)$ ppm. ${ }^{13} \mathrm{C}$ NMR $\left(62.9 \mathrm{MHz}, \mathrm{CDCl}_{3}\right)$ : $\delta=176.3,170.8,70.5,39.0,34.1,32.1,29.8,29.8,29.8,29.8,29.7,29.6$, $29.5,29.5,25.3,22.8,21.2,14.2 \mathrm{ppm}$. IR (film): $\nu_{\max }=3293,3023$, 2925, 2854, 1736, 1716, 1466, 1376, 1242, 1028, $759 \mathrm{~cm}^{-1}$. HRMS (ESI+) $\mathrm{m} / \mathrm{z}$ calcd for $\mathrm{C}_{18} \mathrm{H}_{34} \mathrm{O}_{4} \mathrm{Na}[\mathrm{M}+\mathrm{Na}]^{+}$337.2355, found 337.2352.

4.2.2.10. (R) 3-Acetoxy-heptadecanoic acid ( $7, m=11$ ). Yield $92 \%$. $[\alpha]_{20}^{\mathrm{D}}=-1.7\left(c=1.15, \mathrm{CHCl}_{3}\right) .{ }^{1} \mathrm{H} \mathrm{NMR}\left(250 \mathrm{MHz}, \mathrm{CDCl}_{3}\right): \delta=6.60$ (br s, $\mathrm{COOH}), 5.27-5.13(\mathrm{~m}, 1 \mathrm{H}, \mathrm{CH}-\mathrm{O}), 2.71-2.52\left(\mathrm{~m}, 2 \mathrm{H}, \mathrm{COCH}_{2}\right), 2.04$ $\left(\mathrm{s}, 3 \mathrm{H}, \mathrm{COCH}_{3}\right), 1.73-1.53\left(\mathrm{~m}, 2 \mathrm{H}, \mathrm{CH}-\mathrm{CH}_{2}\right), 1.40-1.16(\mathrm{~m}, 24 \mathrm{H}$, $\left.\left(\mathrm{CH}_{2}\right)_{12} \mathrm{CH}_{3}\right), 0.88\left(\mathrm{t}, 3 \mathrm{H}, \mathrm{CH}_{3}\right)$ ppm. ${ }^{13} \mathrm{C}$ NMR $\left(62.9 \mathrm{MHz}, \mathrm{CDCl}_{3}\right)$ : $\delta=176.1,170.9,70.6,38.9,34.1,32.1,29.8,29.8,29.8,29.8,29.8,29.7$, 29.6, 29.5, 29.5, 25.3, 22.8, 21.2, 14.2 ppm. IR (film): $\nu_{\max }=3300$, 3021, 2926, 2854, 1736, 1715, 1466, 1376, 1246, 1028, $758 \mathrm{~cm}^{-1}$. HRMS (ESI+) $m / z$ calcd for $\mathrm{C}_{19} \mathrm{H}_{36} \mathrm{O}_{4} \mathrm{Na}[\mathrm{M}+\mathrm{Na}]^{+} 351.2511$, found 351.2503.

4.2.2.11. (R) 3-Acetoxy-octadecanoic acid (7, $m=12$ ). Yield 89\%. $[\alpha]_{20}^{\mathrm{D}}=-0.7 \quad\left(c=0.9, \quad \mathrm{CHCl}_{3}\right) .{ }^{1} \mathrm{H} \quad \mathrm{NMR} \quad\left(250 \mathrm{MHz}, \mathrm{CDCl}_{3}\right)$ : $\delta=5.28-5.112(\mathrm{~m}, 1 \mathrm{H}, \mathrm{CH}-\mathrm{O}), 2.70-2.52\left(\mathrm{~m}, 2 \mathrm{H}, \mathrm{COCH}_{2}\right), 2.04(\mathrm{~s}$, $\left.3 \mathrm{H}, \mathrm{COCH}_{3}\right), 1.72-1.53\left(\mathrm{~m}, 2 \mathrm{H}, \mathrm{CH}-\mathrm{CH}_{2}\right), 1.40-1.16(\mathrm{~m}, 26 \mathrm{H}$, $\left.\left(\mathrm{CH}_{2}\right)_{13} \mathrm{CH}_{3}\right), 0.88\left(\mathrm{t}, 3 \mathrm{H}, \mathrm{CH}_{3}\right)$ ppm. ${ }^{13} \mathrm{C}$ NMR $\left(62.9 \mathrm{MHz}, \mathrm{CDCl}_{3}\right)$ : $\delta=175.8,170.7,70.5,38.9,34.1,32.1,29.8,29.8,29.8,29.8,29.8,29.8$, 29.7, 29.6, 29.5, 29.5, 25.3, 22.8, 21.2, 14.3 ppm. IR (film): $\nu_{\max }=3385,3021,2925,2854,1736,1716,1466,1376,1246,1028$, $757 \mathrm{~cm}^{-1}$. HRMS (ESI+) $\mathrm{m} / \mathrm{z}$ calcd for $\mathrm{C}_{20} \mathrm{H}_{38} \mathrm{O}_{4} \mathrm{Na}[\mathrm{M}+\mathrm{Na}]^{+}$ 365.2668, found 365.2663.

4.2.2.12. (R) 3-Acetoxy-nonadecanoic acid ( $7, m=13$ ). Yield $91 \%$. $[\alpha]_{20}^{\mathrm{D}}=-2.4\left(c=0.8, \mathrm{CHCl}_{3}\right) .{ }^{1} \mathrm{H} \mathrm{NMR}\left(250 \mathrm{MHz}, \mathrm{CDCl}_{3}\right): \delta=5.95$ (br s, $\mathrm{COOH}), 5.28-5.12(\mathrm{~m}, 1 \mathrm{H}, \mathrm{CH}-\mathrm{O}), 2.69-2.49\left(\mathrm{~m}, 2 \mathrm{H}, \mathrm{COCH}_{2}\right), 2.04$ $\left(\mathrm{s}, 3 \mathrm{H}, \mathrm{COCH}_{3}\right), 1.71-1.53\left(\mathrm{~m}, 2 \mathrm{H}, \mathrm{CH}-\mathrm{CH}_{2}\right), 1.38-1.16(\mathrm{~m}, 28 \mathrm{H}$, $\left.\left(\mathrm{CH}_{2}\right)_{14} \mathrm{CH}_{3}\right), 0.88\left(\mathrm{t}, 3 \mathrm{H}, \mathrm{CH}_{3}\right)$ ppm. ${ }^{13} \mathrm{C} \mathrm{NMR}\left(62.9 \mathrm{MHz}, \mathrm{CDCl}_{3}\right)$ : $\delta=176.4,170.9,70.5,38.9,34.1,32.1,29.8,29.8,29.8,29.8,29.8,29.8$, $29.8,29.7,29.6,29.5,29.5,25.3,22.8,21.2,14.3$ ppm. IR (film): $\nu_{\max }=3386,3021,2924,2853,1732,1715,1462,1377,1216,1033$,
$758 \mathrm{~cm}^{-1}$. HRMS (ESI+) $\mathrm{m} / z$ calcd for $\mathrm{C}_{21} \mathrm{H}_{40} \mathrm{O}_{4} \mathrm{Na}[\mathrm{M}+\mathrm{Na}]^{+}$ 379.2824, found 379.2826 .

4.2.2.13. (R) 3-Acetoxy-icosanoic acid (7, $m=14$ ). Yield $85 \%$. $[\alpha]_{20}^{\mathrm{D}}=-2.3\left(c=1.45, \mathrm{CHCl}_{3}\right) .{ }^{1} \mathrm{H} \mathrm{NMR}\left(250 \mathrm{MHz}, \mathrm{CDCl}_{3}\right): \delta=5.25-5.11$ (m, 1H, CH-O), 2.69-2.51 (m, 2H, $\left.\mathrm{COCH}_{2}\right), 2.04\left(\mathrm{~s}, 3 \mathrm{H}, \mathrm{COCH}_{3}\right)$, $1.73-1.51\left(\mathrm{~m}, 2 \mathrm{H}, \mathrm{CH}-\mathrm{CH}_{2}\right), 1.37-1.16\left(\mathrm{~m}, 30 \mathrm{H},\left(\mathrm{CH}_{2}\right){ }_{15} \mathrm{CH}_{3}\right), 0.88(\mathrm{t}$, $\left.3 \mathrm{H}, \mathrm{CH}_{3}\right)$ ppm. 13C NMR (62.9 MHz, $\left.\mathrm{CDCl}_{3}\right): \delta=176.4,171.0,70.6,39.0$, 34.1, 32.1, 29.8, 29.8, 29.8, 29.8, 29.8, 29.8, 29.8, 29.8, 29.7, 29.6, 29.5, 29.5, 25.3, 22.8, 21.2, 14.3 ppm. IR (film): $\nu_{\max }=3379,3021,2926$, $2854,1742,1716,1466,1376,1215,1028,758 \mathrm{~cm}^{-1}$. HRMS (ESI+) $\mathrm{m} / z$ calcd for $\mathrm{C}_{22} \mathrm{H}_{42} \mathrm{O}_{4} \mathrm{Na}[\mathrm{M}+\mathrm{Na}]^{+}$393.2981, found 393.2986.

4.2.2.14. (R) 3-Acetoxy-eicosanoic acid (7, $m=15$ ). Yield $89 \%$. $[\alpha]_{20}^{\mathrm{D}}=-1.3\left(c=2.5, \mathrm{CHCl}_{3}\right) .{ }^{1} \mathrm{H} \mathrm{NMR}\left(250 \mathrm{MHz}, \mathrm{CDCl}_{3}\right): \delta=9.20$ (br s, $\mathrm{COOH}$ ), 5.28-5.13 (m, 1H, CH-O), 2.72-2.53 (m, 2H, $\left.\mathrm{COCH}_{2}\right), 2.05$ $\left(\mathrm{s}, 3 \mathrm{H}, \mathrm{COCH}_{3}\right), 1.72-1.52\left(\mathrm{~m}, 2 \mathrm{H}, \mathrm{CH}-\mathrm{CH}_{2}\right), 1.41-1.15(\mathrm{~m}, 32 \mathrm{H}$, $\left.\left(\mathrm{CH}_{2}\right)_{16} \mathrm{CH}_{3}\right), 0.87$ (t, 3H, $\left.\mathrm{CH}_{3}\right)$ ppm. ${ }^{13} \mathrm{C}$ NMR $\left(62.9 \mathrm{MHz}, \mathrm{CDCl}_{3}\right)$ : $\delta=176.6,171.1,70.6,39.0,34.1,32.1,29.8,29.8,29.8,29.8,29.8,29.8$, 29.8, 29.8, 29.8, 29.7, 29.6, 29.5, 29.5, 25.2, 22.8, 21.2, 14.3 ppm. IR (film): $\nu_{\max }=3385,3019,2926,2854,1737,1714,1467,1375,1215$, $1022,757 \mathrm{~cm}^{-1}$. HRMS (ESI+) $\mathrm{m} / z$ calcd for $\mathrm{C}_{23} \mathrm{H}_{44} \mathrm{O}_{4} \mathrm{Na}[\mathrm{M}+\mathrm{Na}]^{+}$ 407.3137, found 407.3134.

4.2.2.15. (R) 3-Acetoxy-docosanoic acid (7, $m=16$ ). Yield 89\%. $[\alpha]_{20}^{\mathrm{D}}=-0.9\left(\mathrm{c}=2.9, \mathrm{CHCl}_{3}\right) .{ }^{1} \mathrm{H}$ NMR $\left(250 \mathrm{MHz}, \mathrm{CDCl}_{3}\right): \delta=9.10$ (br s, $\mathrm{COOH}), 5.29-5.12(\mathrm{~m}, 1 \mathrm{H}, \mathrm{CH}-\mathrm{O}), 2.68-2.52\left(\mathrm{~m}, 2 \mathrm{H}, \mathrm{COCH}_{2}\right), 2.04$ $\left(\mathrm{s}, 3 \mathrm{H}, \mathrm{COCH}_{3}\right), 1.72-1.52\left(\mathrm{~m}, 2 \mathrm{H}, \mathrm{CH}-\mathrm{CH}_{2}\right), 1.41-1.18(\mathrm{~m}, 34 \mathrm{H}$, $\left.\left(\mathrm{CH}_{2}\right)_{17} \mathrm{CH}_{3}\right), 0.87\left(\mathrm{t}, 3 \mathrm{H}, \mathrm{CH}_{3}\right) \mathrm{ppm} .{ }^{13} \mathrm{C}$ NMR $\left(62.9 \mathrm{MHz}, \mathrm{CDCl}_{3}\right)$ : $\delta=176.5,170.8,70.5,39.0,34.1,32.1,29.8,29.8,29.8,29.8,29.8,29.8$, $29.8,29.8,29.8,29.8,29.7,29.6,29.5,29.5,25.2,22.8,21.2,14.2 \mathrm{ppm}$. IR (film): $\nu_{\max }=3358,3021,2925,2854,1736,1716,1465,1376,1215$, $1028,758 \mathrm{~cm}^{-1}$. HRMS (ESI+) $\mathrm{m} / z$ calcd for $\mathrm{C}_{24} \mathrm{H}_{46} \mathrm{O}_{4} \mathrm{Na}[\mathrm{M}+\mathrm{Na}]^{+}$ 421.3294, found 421.3297.

4.2.2.16. (R) 3-Acetoxy-tricosanoic acid (7, $m=17$ ). Yield $85 \%$. $[\alpha]_{20}^{\mathrm{D}}=-1.7\left(c=1.9, \mathrm{CHCl}_{3}\right) .{ }^{1} \mathrm{H}$ NMR $\left(250 \mathrm{MHz}, \mathrm{CDCl}_{3}\right): \delta=7.60$ (br s, $\mathrm{COOH}$ ), 5.27-5.12 (m, 1H, CH-O), 2.69-2.52 (m, 2H, $\left.\mathrm{COCH}_{2}\right), 2.04$ (s, $\left.3 \mathrm{H}, \mathrm{COCH}_{3}\right), 1.72-1.53\left(\mathrm{~m}, 2 \mathrm{H}, \mathrm{CH}-\mathrm{CH}_{2}\right), 1.41-1.15(\mathrm{~m}, 34 \mathrm{H}$, $\left.\left(\mathrm{CH}_{2}\right)_{17} \mathrm{CH}_{3}\right), 0.87\left(\mathrm{t}, 3 \mathrm{H}, \mathrm{CH}_{3}\right) \mathrm{ppm} .{ }^{13} \mathrm{C}$ NMR $\left(62.9 \mathrm{MHz}, \mathrm{CDCl}_{3}\right)$ : $\delta=176.3,170.8,70.5,39.0,34.1,32.1,29.8,29.8,29.8,29.8,29.8,29.8$, $29.8,29.8,29.8,29.8,29.8,29.7,29.6,29.5,29.5,25.3,22.8,21.2$, $14.2 \mathrm{ppm}$. IR (film): $\nu_{\max }=3358,3020,2926,2854,1740,1716,1464$, $1376,1215,1030,757 \mathrm{~cm}^{-1}$. HRMS (ESI+) $\mathrm{m} / z$ calcd for $\mathrm{C}_{25} \mathrm{H}_{48} \mathrm{O}_{4} \mathrm{Na}$ $[\mathrm{M}+\mathrm{Na}]^{+} 435.3450$, found 435.3454 .

4.2.3. Transformation of alcohol $\mathbf{1}(\boldsymbol{R})$ to $\mathbf{3}(\boldsymbol{S})$ with a Mitsunobu reaction. To a solution of alcohol $\mathbf{1}(\boldsymbol{R})(1.21 \mathrm{~g}, 7.03 \mathrm{mmol})$ in toluene $(60 \mathrm{~mL})$ were added $\mathrm{PPh}_{3}(2.75 \mathrm{~g}, 10.5 \mathrm{mmol}, 1.5$ equiv) and acetic acid (1.2 mL, $1.26 \mathrm{~g}, 21 \mathrm{mmol}, 3$ equiv) at room temp. After $10 \mathrm{~min}$, the reaction mixture was cooled to- $30{ }^{\circ} \mathrm{C}$ and diethyl azodicarboxylate ( $1.66 \mathrm{~mL}, 1.83 \mathrm{~g}, 10.5 \mathrm{mmol}, 1.5$ equiv) was added dropwise over a period of $10 \mathrm{~min}$. The reaction mixture was slowly warmed to room temp and stirred for $45 \mathrm{~min}$. It was then filtered and the solvent removed under reduced pressure. The mixture was diluted with saturated $\mathrm{NaHCO}_{3}$ and hexane with vigorous stirring. The layers were separated and the aqueous layer extracted twice with hexane. The combined extracts were dried over $\mathrm{MgSO}_{4}$ and concentrated to give a residue which was purified by silica gel column chromatography (EtOAc/Petroleum ether 1:9) to afford 3 (S) (1.02 g, $4.77 \mathrm{mmol}, 68 \%)$.

$$
3(\boldsymbol{S}):[\alpha]_{20}^{\mathrm{D}}=-5.8\left(c=1.2, \mathrm{CHCl}_{3}\right) .
$$

4.2.3.1. (S) t-Butyl 3-(((R)-3-acetoxy-octanoyl)oxy)pent-4-enoate $\boldsymbol{8}(m=2)$. To a solution of alcohol $\mathbf{1}(\boldsymbol{R})(313 \mathrm{mg}, 1.82 \mathrm{mmol})$ in 
toluene (12 mL) were added $\mathrm{PPh}_{3}$ (908 mg, $3.47 \mathrm{mmol}, 1.9$ equiv) and acid 7 ( $m=2)$ (460 $\mathrm{mg}, 2.28 \mathrm{mmol}, 1.25$ equiv). The reaction mixture was stirred at room temperature. After $15 \mathrm{~min}$, the mixture was cooled down to- $-30^{\circ} \mathrm{C}$ and diethyl azodicarboxylate $(0.57 \mathrm{~mL}$, $604 \mathrm{mg}, 3.47 \mathrm{mmol}, 1.9$ equiv) was added dropwise over a period of $20 \mathrm{~min}$. After complete addition, the reaction mixture was slowly warmed to room temp and stirred for $2 \mathrm{~h}$. The reaction mixture was monitored by TLC. The mixture was filtered and washed with $\mathrm{Et}_{2} \mathrm{O}$. The solvent was concentrated under reduced pressure, to give a residue which was purified by silica gel column chromatography (EtOAc/Petroleum ether, 1:9) to afford 8 ( $m=2$ ) (375 mg, $1.05 \mathrm{mmol}$, $58 \%)$.

Yield 58\%. $[\alpha]_{20}^{\mathrm{D}}=-0.5\left(c=1.0, \mathrm{CHCl}_{3}\right) .{ }^{1} \mathrm{H}$ NMR $\left(250 \mathrm{MHz}, \mathrm{CDCl}_{3}\right)$ : $\delta=5.87-5.70(\mathrm{~m}, 2 \mathrm{H}, 2 \mathrm{CH}=\mathrm{CHaHb}), 5.64-5.56(\mathrm{~m}, 2 \mathrm{H}, 2 \mathrm{CH}-\mathrm{OCO})$, $5.33-5.13\left(\mathrm{~m}, 4 \mathrm{H}, 2 \mathrm{CH}=\mathrm{CH}_{2}\right), 2.65-2.45\left(\mathrm{~m}, 4 \mathrm{H}, 2 \mathrm{COCH}_{2}\right), 2.01(\mathrm{~s}$, $\left.3 \mathrm{H}, \mathrm{OCOCH}_{3}\right), 1.75-1.50\left(\mathrm{~m}, 2 \mathrm{H}, \mathrm{CH}-\mathrm{CH}_{2}\right), 1.43(\mathrm{~s}, 9 \mathrm{H}, t-\mathrm{Bu})$, 1.36-1.20 (m, 6H, $\left.\left(\mathrm{CH}_{2}\right)_{3} \mathrm{CH}_{3}\right), 0.87\left(\mathrm{t}, 3 \mathrm{H}, \mathrm{CH}_{3}\right) \mathrm{ppm} .{ }^{13} \mathrm{C} \mathrm{NMR}$ $\left(62.5 \mathrm{MHz}, \mathrm{CDCl}_{3}\right): \delta=170.4,169.3,168.9,135.1,117.8,81.2,71.5,70.6$, 40.7, 39.3, 34.0, 28.1, 24.9, 22.6, 21.2, $14.1 \mathrm{ppm}$. IR (film): $\nu_{\max }=3020$, 2983, 2926, 2854, 1719, 1597, 1467, 1370, 1292, 1216, 1155, 1107, $1024,935,837,755 \mathrm{~cm}^{-1}$. HRMS (ESI+) $\mathrm{m} / z$ calcd for $\mathrm{C}_{19} \mathrm{H}_{32} \mathrm{O}_{6} \mathrm{Na}$ $[\mathrm{M}+\mathrm{Na}]^{+}$379.2097, found 379.2088.

4.2.4. General procedure for the cross-metathesis and hydrogenation, access to $\mathbf{9 a}-\boldsymbol{e}(n=2$ to $n=6)$. To a solution of $\mathbf{8}(m=2)(0.2 \mathrm{mmol})$ in anhydrous ethyl ether $(5 \mathrm{~mL})$ were added Grubbs2 catalyst (10.2 mg, $12 \mu \mathrm{mol}, 6 \mathrm{~mol} \%$ ), CuI (3.4 mg, $18 \mu \mathrm{mol}, 9 \%$ eq) and alkene (1.2 mmol, 6 equiv) under an Ar atmosphere. The mixture was heated to $40{ }^{\circ} \mathrm{C}$ for $2 \mathrm{~h}$ until the color of the reaction mixture turned from brown to dark green, corresponding to disappearance of starting material by TLC. After cooling to room temperature, the mixture was filtered through a short column of sand and $\mathrm{MgSO}_{4}$. The solid was washed with ethyl ether and the solvent was evaporated under reduced pressure. The obtained residue was used for the next step without further purification. To a solution of residue in dry EtOAc $(5 \mathrm{~mL})$ at room temperature, a catalytic amount of $\mathrm{PtO}_{2}$ was added. The flask was purged and filled with $\mathrm{H}_{2}$ gas. The mixture was stirred vigorously at room temperature for $2 \mathrm{~h}$. The reaction was filtered through a Celite pad and washed with EtOAc. The solvent was removed and the crude product was purified by silica gel column chromatography (EtOAc/Petroleum ether as eluent, $1: 19)$ to afford the corresponding adduct $9 \mathbf{a}-\mathbf{e}(n=2$ to $n=6)$ (48-60\% yield for two steps).

4.2.4.1. (R) t-Butyl 3-(((R)-3-acetoxy-octanoyl)oxy)octanoate 9a $(n=2)$. Yield $60 \% .[\alpha]_{20}^{\mathrm{D}}=-1.2\left(c=1.0, \mathrm{CHCl}_{3}\right) .{ }^{1} \mathrm{H}$ NMR $(500 \mathrm{MHz}$, $\left.\mathrm{CDCl}_{3}\right): \delta=5.22-5.17(\mathrm{~m}, 2 \mathrm{H}, 2 \mathrm{CH}-\mathrm{O}), 2.59-2.41\left(\mathrm{~m}, 4 \mathrm{H}, \alpha-\mathrm{CH}_{2}, \alpha^{\prime}-\right.$ $\left.\mathrm{CH}_{2}\right), 2.02\left(\mathrm{~s}, 3 \mathrm{H}, \mathrm{COCH}_{3}\right), 1.65-1.51\left(\mathrm{~m}, 4 \mathrm{H}, 2 \mathrm{CH}_{2}\right), 1.43(\mathrm{~s}, 9 \mathrm{H}, t-\mathrm{Bu})$, $1.35-1.20\left(\mathrm{~m}, 12 \mathrm{H}, 6 \mathrm{CH}_{2}\right), 0.87\left(\mathrm{t}, 6 \mathrm{H}, 2 \mathrm{CH}_{3}\right)$ ppm. ${ }^{13} \mathrm{C} \mathrm{NMR}$ $\left(125 \mathrm{MHz}, \mathrm{CDCl}_{3}\right): \delta=170.5,169.8,169.7,81.0,71.3,70.7,40.6,39.3$, $34.2,34.0,31.7,31.7,28.2,25.2,24.9,22.8,22.6,21.2,14.2,14.1 \mathrm{ppm}$. IR (film): $\nu_{\max }=3020,2928,2856,1738,1463,1369,1242,1159,1026$, $757 \mathrm{~cm}^{-1}$. HRMS (ESI+) $\mathrm{m} / \mathrm{z}$ calcd for $\mathrm{C}_{22} \mathrm{H}_{40} \mathrm{O}_{6} \mathrm{Na}[\mathrm{M}+\mathrm{Na}]^{+}$ 423.2723, found 423.2729 .

4.2.4.2. (R) t-Butyl 3-(((R)-3-acetoxy-octanoyl)oxy)nonanoate $\mathbf{9 b}$ $(n=3)$. Yield 53\%. $[\alpha]_{20}^{\mathrm{D}}=+2.2\left(c=1.75, \mathrm{CHCl}_{3}\right) .{ }^{1} \mathrm{H} \mathrm{NMR}(600 \mathrm{MHz}$, $\left.\mathrm{CDCl}_{3}\right): \delta=5.22-5.15(\mathrm{~m}, 2 \mathrm{H}, 2 \mathrm{CH}-\mathrm{O}), 2.59-2.40\left(\mathrm{~m}, 4 \mathrm{H}, \alpha-\mathrm{CH}_{2}, \alpha^{\prime}-\right.$ $\left.\mathrm{CH}_{2}\right), 2.01\left(\mathrm{~s}, 3 \mathrm{H}, \mathrm{COCH}_{3}\right), 1.63-1.51\left(\mathrm{~m}, 4 \mathrm{H}, 2 \mathrm{CH}_{2}\right), 1.42(\mathrm{~s}, 9 \mathrm{H}, t-\mathrm{Bu})$, 1.35-1.20 (m, 14H, $\left.7 \mathrm{CH}_{2}\right), 0.87\left(\mathrm{t}, 6 \mathrm{H}, 2 \mathrm{CH}_{3}\right)$ ppm. ${ }^{13} \mathrm{C} \mathrm{NMR}$ $\left(150 \mathrm{MHz}, \mathrm{CDCl}_{3}\right): \delta=170.4,169.8,169.7,80.9,71.3,70.6,40.6,39.3$, $34.1,33.9,31.8,31.7,29.2,28.1,25.2,24.9,22.7,22.6,21.2,14.2$, $14.1 \mathrm{ppm}$. IR (film): $\nu_{\max }=3023,2961,2861,1736,1461,1369,1244$, $1161,1027,972,758 \mathrm{~cm}^{-1}$. HRMS (ESI+) $\mathrm{m} / z$ calcd for $\mathrm{C}_{23} \mathrm{H}_{42} \mathrm{O}_{6} \mathrm{Na}$ $[\mathrm{M}+\mathrm{Na}]^{+}$437.2879, found 437.2873.
4.2.4.3. (R) t-Butyl 3-(((R)-3-acetoxy-octanoyl)oxy)decanoate 9c $(n=4)$. Yield $48 \% .[\alpha]_{20}^{\mathrm{D}}=+2.9\left(c=1.5, \mathrm{CHCl}_{3}\right) .{ }^{1} \mathrm{H}$ NMR $(500 \mathrm{MHz}$, $\left.\mathrm{CDCl}_{3}\right): \delta=5.22-5.17(\mathrm{~m}, 2 \mathrm{H}, 2 \mathrm{CH}-\mathrm{O}), 2.59-2.40\left(\mathrm{~m}, 4 \mathrm{H}, \alpha-\mathrm{CH}_{2}, \alpha^{\prime}-\right.$ $\left.\mathrm{CH}_{2}\right), 2.02\left(\mathrm{~s}, 3 \mathrm{H}, \mathrm{COCH}_{3}\right), 1.63-1.52\left(\mathrm{~m}, 4 \mathrm{H}, 2 \mathrm{CH}_{2}\right), 1.43(\mathrm{~s}, 9 \mathrm{H}, t-$ $\mathrm{Bu}), 1.34-1.22\left(\mathrm{~m}, 16 \mathrm{H}, 8 \mathrm{CH}_{2}\right), 0.87\left(\mathrm{t}, 6 \mathrm{H}, 2 \mathrm{CH}_{3}\right) \mathrm{ppm} .{ }^{13} \mathrm{C} \mathrm{NMR}$ $\left(125 \mathrm{MHz}, \mathrm{CDCl}_{3}\right): \delta=170.5,169.8,169.7,81.0,71.3,70.7,40.6,39.4$, $34.1,34.0,31.9,31.7,29.5,29.3,28.2,25.2,24.9,22.8,22.6,21.3,14.2$, $14.1 \mathrm{ppm}$ IR (film): $\nu_{\max }=3022,2928,2857,1736,1458,1368,1243$, 1157, 1026, $757 \mathrm{~cm}^{-1}$. HRMS (ESI+) $\mathrm{m} / z$ calcd for $\mathrm{C}_{24} \mathrm{H}_{44} \mathrm{O}_{6} \mathrm{Na}$ $[\mathrm{M}+\mathrm{Na}]^{+}$451.3036, found 451.3028 .

4.2.4.4. (R) t-Butyl 3-(((R)-3-acetoxy-octanoyl)oxy)undecanoate 9d $(n=5)$. Yield $54 \%$. $[\alpha]_{20}^{\mathrm{D}}=+2.6\left(c=2.2, \mathrm{CHCl}_{3}\right) .{ }^{1} \mathrm{H} \mathrm{NMR}(600 \mathrm{MHz}$, $\left.\mathrm{CDCl}_{3}\right): \delta=5.21-5.16(\mathrm{~m}, 2 \mathrm{H}, 2 \mathrm{CH}-\mathrm{O}), 2.59-2.40\left(\mathrm{~m}, 4 \mathrm{H}, \alpha-\mathrm{CH}_{2}, \alpha^{\prime}-\right.$ $\left.\mathrm{CH}_{2}\right), 2.01\left(\mathrm{~s}, 3 \mathrm{H}, \mathrm{COCH}_{3}\right), 1.62-1.52\left(\mathrm{~m}, 4 \mathrm{H}, 2 \mathrm{CH}_{2}\right), 1.42(\mathrm{~s}, 9 \mathrm{H}, t-$ $\mathrm{Bu}), 1.34-1.20\left(\mathrm{~m}, 18 \mathrm{H}, 9 \mathrm{CH}_{2}\right), 0.86\left(\mathrm{t}, 6 \mathrm{H}, 2 \mathrm{CH}_{3}\right) \mathrm{ppm} .{ }^{13} \mathrm{C} \mathrm{NMR}$ $\left(150 \mathrm{MHz}, \mathrm{CDCl}_{3}\right): \delta=170.4,169.8,169.7,80.9,71.2,70.6,40.6,39.3$, 34.1, 34.0, 32.0, 31.7, 29.6, 29.5, 29.3, 28.1, 25.2, 24.9, 22.8, 22.6, 21.2, $14.2,14.1 \mathrm{ppm}$. IR (film): $\nu_{\max }=3022,2928,2856,1735,1458,1369$, $1246,1216,1156,1027,955,757 \mathrm{~cm}^{-1}$. HRMS (ESI+) $\mathrm{m} / z$ calcd for $\mathrm{C}_{25} \mathrm{H}_{46} \mathrm{O}_{6} \mathrm{Na}[\mathrm{M}+\mathrm{Na}]^{+}$465.3192, found 465.3199 .

4.2.4.5. (R) t-Butyl 3-(((R)-3-acetoxy-octanoyl)oxy)dodecanoate 9e $(n=6)$. Yield $54 \% .[\alpha]_{20}^{\mathrm{D}}=+3.2\left(c=1.5, \mathrm{CHCl}_{3}\right) .{ }^{1} \mathrm{H}$ NMR $(250 \mathrm{MHz}$, $\left.\mathrm{CDCl}_{3}\right): \delta=5.24-5.14(\mathrm{~m}, 2 \mathrm{H}, 2 \mathrm{CH}-\mathrm{O}), 2.62-2.37\left(\mathrm{~m}, 4 \mathrm{H}, \alpha-\mathrm{CH}_{2}, \alpha^{\prime}-\right.$ $\left.\mathrm{CH}_{2}\right), 2.01\left(\mathrm{~s}, 3 \mathrm{H}, \mathrm{COCH}_{3}\right), 1.67-1.50\left(\mathrm{~m}, 4 \mathrm{H}, 2 \mathrm{CH}_{2}\right), 1.42(\mathrm{~s}, 9 \mathrm{H}, t-$ $\mathrm{Bu}), 1.36-1.19\left(\mathrm{~m}, 20 \mathrm{H}, 10 \mathrm{CH}_{2}\right), 0.86\left(\mathrm{t}, 6 \mathrm{H}, 2 \mathrm{CH}_{3}\right) \mathrm{ppm} .{ }^{13} \mathrm{C} \mathrm{NMR}$ $\left(62.5 \mathrm{MHz}, \mathrm{CDCl}_{3}\right): \delta=170.4,169.8,169.7,81.0,71.3,70.6,40.6,39.3$, 34.1, 34.0, 32.0, 31.7, 29.6, 29.6, 29.5, 29.4, 28.1, 25.2, 24.9, 22.8, 22.6, $21.2,14.2,14.1 \mathrm{ppm}$. IR (film): $\nu_{\max }=3025,2929,2859,1736,1459$, $1368,1242,1161,1027,972,757 \mathrm{~cm}^{-1}$. HRMS (ESI+) $\mathrm{m} / z$ calcd for $\mathrm{C}_{26} \mathrm{H}_{48} \mathrm{O}_{6} \mathrm{Na}[\mathrm{M}+\mathrm{Na}]^{+}$479.3349, found 479.3340 .

4.2.5. General procedure for the final deprotection, same as access to compounds 7

4.2.5.1. (R) 3-((R)-3-Acetoxy-octanoyl)oxy)octanoic acid 10a $(n=2)$. Yield 76\%. $[\alpha]_{20}^{\mathrm{D}}=-1.0\left(c=0.25, \mathrm{CHCl}_{3}\right) .{ }^{1} \mathrm{H}$ NMR $(600 \mathrm{MHz}$, $\left.\mathrm{CDCl}_{3}\right): \delta=5.24-5.16(\mathrm{~m}, 2 \mathrm{H}, 2 \mathrm{CH}-\mathrm{O}), 2.66-2.50\left(\mathrm{~m}, 4 \mathrm{H}, \alpha-\mathrm{CH}_{2}\right.$, $\left.\alpha^{\prime}-\mathrm{CH}_{2}\right), 2.02\left(\mathrm{~s}, 3 \mathrm{H}, \mathrm{COCH}_{3}\right), 1.68-1.52\left(\mathrm{~m}, 4 \mathrm{H}, 2 \mathrm{CH}_{2}\right), 1.38-1.19$ $\left(\mathrm{m}, 12 \mathrm{H}, 6 \mathrm{CH}_{2}\right), 0.87\left(\mathrm{t}, 6 \mathrm{H}, 2 \mathrm{CH}_{3}\right) \mathrm{ppm} .{ }^{13} \mathrm{C} \mathrm{NMR}(150 \mathrm{MHz}$, $\left.\mathrm{CDCl}_{3}\right): \delta=175.6,170.6,169.9,70.8,70.7,39.4,38.4,34.0,33.9,31.7$, 31.6, 24.9, 22.6, 21.2, $14.1 \mathrm{ppm}$. IR (film): $\nu_{\max }=2958,2927,2856$, $1745,1715,1433,1376,1238,1172,1124,1026,805 \mathrm{~cm}^{-1}$. HRMS (ESI+) $\mathrm{m} / \mathrm{z}$ calcd for $\mathrm{C}_{18} \mathrm{H}_{32} \mathrm{O}_{6} \mathrm{Na}[\mathrm{M}+\mathrm{Na}]^{+}$367.2097, found 367.2079 .

4.2.5.2. (R) 3-(((R)-3-Acetoxy-octanoyl)oxy)nonanoic acid $\mathbf{1 0 b}$ $(n=3)$. Yield $86 \% .[\alpha]_{20}^{\mathrm{D}}=+1.1\left(c=0.9, \mathrm{CHCl}_{3}\right) .{ }^{1} \mathrm{H}$ NMR $(500 \mathrm{MHz}$, $\left.\mathrm{CDCl}_{3}\right): \delta=5.75(\mathrm{br} \mathrm{s}, 1 \mathrm{H}, \mathrm{OH}), 5.26-5.15(\mathrm{~m}, 2 \mathrm{H}, 2 \mathrm{CH}-\mathrm{O})$, 2.66-2.49 (m, 4H, $\left.\alpha-\mathrm{CH} 2, \alpha^{\prime}-\mathrm{CH}_{2}\right), 2.02\left(\mathrm{~s}, 3 \mathrm{H}, \mathrm{COCH}_{3}\right), 1.69-1.50$ (m, 4H, $\left.2 \mathrm{CH}_{2}\right), 1.38-1.18\left(\mathrm{~m}, 14 \mathrm{H}, 7 \mathrm{CH}_{2}\right), 0.87\left(\mathrm{t}, 6 \mathrm{H}, 2 \mathrm{CH}_{3}\right) \mathrm{ppm}$. ${ }^{13} \mathrm{C}$ NMR $\left(125 \mathrm{MHz}, \mathrm{CDCl}_{3}\right): \delta=175.3,170.6,169.9,70.9,70.7,39.4$, $38.8,34.0,31.8,31.7,29.1,25.2,24.9,22.7,22.6,21.2,14.2,14.1 \mathrm{ppm}$. IR (film): $\nu_{\max }=2965,2928,2859,1745,1714,1435,1378,1240,1172$, $1121,1027,806,725 \mathrm{~cm}^{-1}$. HRMS (ESI+) $\mathrm{m} / z$ calcd for $\mathrm{C}_{19} \mathrm{H}_{34} \mathrm{O}_{6} \mathrm{Na}$ $[\mathrm{M}+\mathrm{Na}]^{+}$381.2253, found 381.2249.

4.2.5.3. (R) 3-(((R)-3-Acetoxy-octanoyl)oxy)decanoic acid 10c $(n=4)$. Yield 70\%. $[\alpha]_{20}^{\mathrm{D}}=-1.4\left(c=0.85, \mathrm{CHCl}_{3}\right) .{ }^{1} \mathrm{H} \mathrm{NMR}(600 \mathrm{MHz}$, $\mathrm{CDCl}_{3}$ ): $\delta=7.38$ (br s, $\left.1 \mathrm{H}, \mathrm{OH}\right), 5.25-5.15(\mathrm{~m}, 2 \mathrm{H}, 2 \mathrm{CH}-\mathrm{O}), 2.66-2.50$ $\left(\mathrm{m}, 4 \mathrm{H}, \alpha-\mathrm{CH}_{2}, \alpha^{\prime}-\mathrm{CH}_{2}\right), 2.02\left(\mathrm{~s}, 3 \mathrm{H}, \mathrm{COCH}_{3}\right), 1.68-1.50(\mathrm{~m}, 4 \mathrm{H}, 2$ $\left.\mathrm{CH}_{2}\right), 1.38-1.20\left(\mathrm{~m}, 16 \mathrm{H}, 8 \mathrm{CH}_{2}\right), 0.87\left(\mathrm{t}, 6 \mathrm{H}, 2 \mathrm{CH}_{3}\right) \mathrm{ppm} .{ }^{13} \mathrm{C} \mathrm{NMR}$ $\left(150 \mathrm{MHz}, \mathrm{CDCl}_{3}\right): \delta=175.9,170.8,170.0,70.9,70.8,39.4,38.8,34.0$, $31.9,31.7,29.4,29.3,25.2,24.9,22.7,22.6,21.2,14.2,14.1 \mathrm{ppm}$. IR (film): $\nu_{\max }=2963,2928,2858,1744,1712,1434,1377,1240,1171$, 
$1123,1027,953,725 \mathrm{~cm}^{-1}$. HRMS (ESI+) $m / z$ calcd for $\mathrm{C}_{20} \mathrm{H}_{36} \mathrm{O}_{6} \mathrm{Na}$ $[\mathrm{M}+\mathrm{Na}]^{+}$395.2410, found 395.2404.

4.2.5.4. (R) 3-(((R)-3-Acetoxy-octanoyl)oxy)undecanoic acid 10d $(n=5)$. Yield $73 \%$. $[\alpha]_{20}^{\mathrm{D}}=+2.2\left(c=1.25, \mathrm{CHCl}_{3}\right) .{ }^{1} \mathrm{H}$ NMR $(600 \mathrm{MHz}$, $\mathrm{CDCl}_{3}$ ): $\delta=8.0$ (br s, $1 \mathrm{H}, \mathrm{OH}$ ), 5.25-5.16 (m, 2H, $2 \mathrm{CH}-0$ ), 2.66-2.50 $\left(\mathrm{m}, 4 \mathrm{H}, \alpha-\mathrm{CH}_{2}, \alpha^{\prime}-\mathrm{CH}_{2}\right), 2.02\left(\mathrm{~s}, 3 \mathrm{H}, \mathrm{COCH}_{3}\right), 1.68-1.51(\mathrm{~m}, 4 \mathrm{H}, 2$ $\left.\mathrm{CH}_{2}\right), 1.38-1.19\left(\mathrm{~m}, 18 \mathrm{H}, 9 \mathrm{CH}_{2}\right), 0.87\left(\mathrm{t}, 6 \mathrm{H}, 2 \mathrm{CH}_{3}\right) \mathrm{ppm} .{ }^{13} \mathrm{C} \mathrm{NMR}$ $\left(150 \mathrm{MHz}, \mathrm{CDCl}_{3}\right): \delta=175.9,170.7,169.9,70.9,70.7,39.4,38.8,34.0$, $31.9,31.7,29.6,29.5,29.3,25.2,24.8,22.8,22.6,21.2,14.2,14.1$ ppm. IR (film): $\nu_{\max }=2961,2926,2856,1743,1710,1457,1377,1240,1172$, $1123,1027,801 \mathrm{~cm}^{-1}$. HRMS (ESI+) $\mathrm{m} / z$ calcd for $\mathrm{C}_{21} \mathrm{H}_{38} \mathrm{O}_{6} \mathrm{Na}$ $[\mathrm{M}+\mathrm{Na}]^{+}$409.2566, found 409.2570.

4.2.5.5. (R) 3-(((R)-3-Acetoxy-octanoyl)oxy)dodecanoic acid 10e $(n=6)$. Yield $88 \%$. $[\alpha]_{20}^{\mathrm{D}}=-1.6\left(c=0.65, \mathrm{CHCl}_{3}\right) .{ }^{1} \mathrm{H}$ NMR $(600 \mathrm{MHz}$, $\left.\mathrm{CDCl}_{3}\right): \delta=5.25-5.16(\mathrm{~m}, 2 \mathrm{H}, 2 \mathrm{CH}-\mathrm{O}), 2.66-2.49\left(\mathrm{~m}, 4 \mathrm{H}, \alpha-\mathrm{CH}_{2}, \alpha^{\prime}-\right.$ $\left.\mathrm{CH}_{2}\right), 2.02\left(\mathrm{~s}, 3 \mathrm{H}, \mathrm{COCH}_{3}\right), 1.68-1.50\left(\mathrm{~m}, 4 \mathrm{H}, 2 \mathrm{CH}_{2}\right), 1.38-1.19(\mathrm{~m}$, $\left.20 \mathrm{H}, 10 \mathrm{CH}_{2}\right), 0.87\left(\mathrm{t}, 6 \mathrm{H}, 2 \mathrm{CH}_{3}\right) \mathrm{ppm} .{ }^{13} \mathrm{C} \mathrm{NMR}\left(150 \mathrm{MHz}, \mathrm{CDCl}_{3}\right)$ : $\delta=175.4,170.6,169.9,70.9,70.7,39.4,38.8,34.0,32.0,31.7,29.7$, 29.6, 29.5, 29.4, 25.2, 24.9, 22.8, 22.6, 21.2, 14.2, 14.1 ppm. IR (film): $\nu_{\max }=2960,2925,2855,1742,1709,1457,1375,1239,1171,1123$, 1026, $799 \mathrm{~cm}^{-1}$. HRMS (ESI +$) \mathrm{m} / z$ calcd for $\mathrm{C}_{22} \mathrm{H}_{40} \mathrm{O}_{6} \mathrm{Na}[\mathrm{M}+\mathrm{Na}]^{+}$ 423.2712, found 423.2723 .

\section{Acknowledgements}

Financial support from the Centre National de la Recherche Scientifique, the Ministry of Higher Education and Research (MESR) and from the ARC grant 'Field', financed by the French Community of Belgium are gratefully acknowledged. Magali Deleu thanks the Fonds National de la Recherche Scientifique (FNRS) from Belgium for her Senior Research Associate position.

\section{Supplementary data}

Supplementary data (Copies of the ${ }^{1} \mathrm{H}$ and ${ }^{13} \mathrm{C}$ NMR spectra, FTIR and HRMS spectra for all new compounds) associated with this article can be found in the online version, at http://dx.doi.org/ $10.1016 / j . t e t .2016 .09 .065$.

\section{References and notes}

1. (a) Mitsunobu, O.; Yamada, M. Bull. Chem. Soc. Jpn. 1967, 40, 2380-2382; (b) Mitsunobu, O. Synthesis 1981, 1,1-28; (c) Kumara Swamy, K. C.; Bhuvan Kumar,
N. N.; Balaraman, E.; Pavan Kumar, K. V. P. Chem. Rev. 2009, 109, 2551-2651; (d) Fletcher, S. Org. Chem. Front. 2015, 2, 739-752.

2. (a) Myers, A. G.; Glatthar, R.; Hammond, M.; Harrington, P. M.; Kuo, E. Y.; Liang J.; Schaus, S. E.; Wu, Y.; Xiang, J.-N. J. Am. Chem. Soc. 2002, 124, 5380-5401; (b) Shen, R.; Lin, C. T.; Porco, J. A., Jr. J. Am. Chem. Soc. 2002, 124, 5650-5651; (c) Wipf, P. Reeves, J. T. Chem. Commun. 2002, 2066-2067; (d) Shen, R.; Lin, C. T. Bowman, E. J.; Bowman, B. J.; Porco, J. A., Jr. J. Am. Chem. Soc. 2003, 125, 7889-7901; (e) Chen, Y.; Gambs, C.; Abe, Y.; Wentworth, P., Jr.; Janda, K. D. J. Org. Chem. 2003, 68, 8902-8905; (f) Paterson, I.; Tudge, M. Tetrahedron 2003. 59, 6833-6849; (g) Zhu, L.; Kedenburg, J. P.; Xian, M.; Wang, P. G. Tetrahedron Lett. 2005, 46, 811-813; (h) Suenaga, K.; Hoshino, H.; Yoshii, T.; Mori, K.; Sone, H.; Bessho, Y.; Sakakura, A.; Hayakawa, I.; Yamada, K.; Kigoshi, H. Tetrahedron 2006, 62, 7687-7698; (i) Van Orden, L. J. Patterson, B. D. Rychnovsky, S. D. J. Org. Chem. 2007, 72, 5784-5793; (j) Sharma, A.; Gamre, S.; Roy, S.; Goswami, D.; Chattopadhyay, A.; Chattopadhyay, S. Tetrahedron Lett. 2008, 49, 3902-3905; (k) Snaddon, T. N.; Buchgraber, P.; Schulthoff, S.; Wirtz, C.: Mynott, R.; Fürstner, A. Chem.—Eur. J. 2010, 16, 12133-12140; (1) Lu, H.-H.; Hinkelmann, B.; Tautz, T.; Li, J.; Sasse, F.; Franke, R.; Kalesse, M. Org. Biomol. Chem. 2015, 13, 8029-8036.

3. (a) Hodgson, D. M.: Gibbs, A. R. Synlett 1997, 657-658; (b) Hodgson, D. M.; Gibbs, A. R.; Drew, M. G. J. Chem. Soc., Perkin Trans. 1 1999, 3579-3590; (c) Tietze, L. F.; Stadler, C.; Böhnke, N.; Brasche, G.; Grube, A. Synlett 2007, 485-487; (d) Enev, V. S.; Drescher, M.; Mulzer, J. Tetrahedron 2007, 63, 5930-5939; (e) Enev, V. S.; Felzmann, W.; Gromov, A.; Marchart, S.; Mulzer, J. Chem.-Eur. J. 2012, 18, 9651-9668.

4. (a) Abdel-Mawgoud, A. M.; Lépine, F.; Déziel, E. Appl. Microbiol. Biotechnol. 2010, 86,1323-1336 and references cited therein; (b) Menhour, B.; Mayon, P.: Plé, K.; Bouquillon, S.; Dorey, S.; Clément, C.; Deleu, M.; Haudrechy, A. Tetrahedron Lett. 2015, 56, 1159-1161.

5. (a) Jarvis, F. G.; Johnson, M. J. J. Am. Chem. Soc. 1949, 71, 4124-4126; (b) Hauser, G.; Karnovsky, M. L. J. Bacteriol. 1954, 68, 645-655; (c) Edwards, J. R.; Hayashi, J. A. Arch. Biochem. Biophys. 1965, 111, 415-421; (d) Johnson, M. K.; Boese-Marazzo, D. Infect. Immun. 1980, 29, 1028-1033; (e) Hirayama, T.; Kato, I. FEBS Lett. 1982, 139, 81-85; (f) Syldatk, C.; Lang, S.; Wagner, F.; Wray, V.; Witte, L. Z. Naturforsch., Teil C 1985, 40, 51-60.

6. (a) Häussler, S.; Nimtz, M.; Domke, T.; Wray, V.; Steinmetz, I. Infect. Immun. 1998, 66, 1588-1593; (b) Andrä, J.; Rademann, J.; Howe, J.; Koch, M. H. J.; Heine, H.; Zähringer, U.; Brandenburg, K. Biol. Chem. 2006, 387, 301-310.

7. (a) Varnier, A. L.; Sanchez, L.; Vatsa, P.; Boudesocque, L.; Garcia-Brugger, A.; Rabeoelina, F.; Sorokin, A.; Renault, J.-H.; Kauffmann, S.; Pugin, A.; Clément, C.; Baillieul, F: Dorey, S. Plant Cell Environ. 2009, 32, 178-193; (b) Vatsa, P. Sanchez, L.; Clément, C.; Baillieul, F.; Dorey, S. Int. J. Mol. Sci. 2010, 11, 5095-5108; (c) Sanchez, L.; Courteaux, B.; Hubert, J.; Kauffmann, S.; Renault, J.-H.; Clément, C.; Baillieul, F.; Dorey, S. Plant Physiol. 2012, 160 1630-1441; (d) Delaunois, B.; Farace, G.; Jeandet, P.; Clément, C.; Baillieul, F.; Dorey, S.; Cordelier, S. Environ. Sci. Pollut. Res. 2014, 21, 4837-4846.

8. Duynstee, H. I.; Van Vliet, M. J.; van der Marel, G. A.; van Boom, J. H. Eur. J. Org. Chem. 1998, 2, 303-307.

9. (a) Tan, C.-H.; Holmes, A. B. Chem.-Eur. J. 2001, 7, 1845-1854; (b) Souto, J. A.; Vaz, E.; Lepore, I.; Pöppler, A.-C.; Franci, G.; Alvarez, R.; Altucci, L.; de Lera, A. R. J. Med. Chem. 2010, 53, 4654-4667; (c) Kaji, E.; Komori, T.; Yokoyama, M.; Kato, T.; Nishino, T.; Shirahata, T. Tetrahedron 2010, 66, 4089-4100.

10. This reaction has been performed on more than five grams, with a $94 \%$ ee, measured by chiral HPLC with an IC column.

11. Wohlrab, A.; Lamer, R.; VanNieuwenhze, M. S. J. Am. Chem. Soc. 2007, 129, 4175-4177.

12. Voigtritter, K.; Ghorai, S.; Lipshutz, B. H. J. Org. Chem. 2011, 76, 4697-4702. 